\title{
TOWARD UNDERSTANDING THE B[e] PHENOMENON. II. NEW GALACTIC FS CMa STARS ${ }^{1}$
}

\author{
A. S. Miroshnichenko \\ Department of Physics and Astronomy, University of North Carolina at Greensboro, Greensboro, NC 27402; a_mirosh@uncg.edu \\ N. Manset \\ CFHT Corporation, 65-1238 Mamalahoa Highway, Kamuela, HI 96743 \\ A. V. KUSAKIN \\ Sternberg Astronomical Institute, Universitetskij pr. 13, Moscow, Russia, Fesenkov Astrophysical Institute, \\ Kamenskoe plato, Almaty 050020, Kazakhstan \\ E. L. Chentsov and V. G. Klochkova \\ Special Astrophysical Observatory of the Russian Academy of Sciences, Nizhnyj Arkhyz, 369167, Russia \\ S. V. ZHARIKOV \\ Instituto de Astronomía, Universidad Nacional Autónoma de México, Apartado Postal 877, 22830, Ensenada, Baja California, México \\ R. O. GRAY \\ Department of Physics and Astronomy, Appalachian State University, Boone, NC 28608 \\ K. N. Grankin \\ Ulugh Beg Astronomical Institute, Astronomicheskaya St. 33, Tashkent 700052, Uzbekistan \\ T. L. GANDET \\ Lizard Hollow Observatory, P.O. Box 89175, Tucson, AZ 85752-9175 \\ K. S. BJORKMAN \\ Ritter Observatory, University of Toledo, Toledo, OH 43606-3390
}

R. J. Rudy, D. K. Lynch, C. C. Venturini, and S. Mazuk

The Aerospace Corporation, M2/266, P.O. Box 92957, Los Angeles, CA 90009

R. C. PuetTer

University of California, San Diego, 9500 Gilman Dr., La Jolla, CA 92093

R. B. PERRY

Science Support Office, M/S 160, NASA Langley Research Center, Hampton, VA 23681

H. Levato and M. Grosso

Complejo Astronómico El Leoncito (CASLEO) Casilla de Correo 467, 5400, San Juan, Argentina

S. BERNABEI

INAF-Osservatorio Astronomico di Bologna, Via Ranzani 1, 40127 Bologna, Italy

V. F. Polcaro and R. F. Viotti

Istituto di Astrofisica Spaziale e Fisica Cosmica, INAF, Via del Fosso del Cavaliere 100, 00133, Roma, Italy

L. NORCI

School of Physical Sciences and NCPST, Dublin City University, Glasnevin, Dublin 9, Ireland

AND

K. S. Kuratov

Fesenkov Astrophysical Institute, Kamenskoe plato, Almaty 050020, Kazakhstan Received 2007 May 25; accepted 2007 August 31

\section{ABSTRACT}

FS CMa stars form a group of objects with the $\mathrm{B}[\mathrm{e}]$ phenomenon that were previously known as unclassified $\mathrm{B}[\mathrm{e}]$ stars or $\mathrm{B}[\mathrm{e}]$ stars with warm dust $(\mathrm{B}[\mathrm{e}] \mathrm{WD})$ until recently. They exhibit strong emission-line spectra and strong IR excesses, most likely due to recently formed circumstellar dust. These properties have been suggested to be due to ongoing or recent rapid mass exchange in binary systems with hot primaries and various types of secondaries. The first paper of this series reported an analysis of the available information about previously known Galactic objects with the $\mathrm{B}[\mathrm{e}]$ phenomenon, the initial selection of the FS CMa group objects, and a qualitative explanation of their properties. This paper reports the results of our new search for more FS CMa objects in the IRAS Point Source Catalog. We present new photometric criteria for identifying FS CMa stars as well as the first results of our observations of nine new FS CMa group members. With this addition, the FS CMa group has now 40 members, becoming

\footnotetext{
1 Partially based on data obtained at the $6 \mathrm{~m}$ BTA Telescope of the Russian Academy of Sciences, $3.6 \mathrm{~m}$ Canada-France-Hawaii Telescope, $3 \mathrm{~m}$ Shane Telescope of the

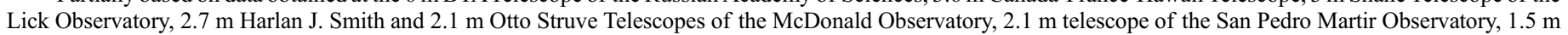
telescope of the Loiano Observatory, and $0.8 \mathrm{~m}$ telescope of the Dark Sky Observatory.
} 
the largest among the dust-forming hot star groups. We also present nine objects with no evidence for the $\mathrm{B}[\mathrm{e}] \mathrm{phe}-$ nomenon, but with newly discovered spectral line emission and/or strong IR excesses.

Subject headings: circumstellar matter — stars: early-type — stars: emission-line, Be

\section{INTRODUCTION}

The $\mathrm{B}[\mathrm{e}]$ phenomenon was first recognized by Allen \& Swings (1976), who found a group of 65 Galactic emission-line B-type stars with large IR excesses due to CS dust. The objects' optical spectra contain forbidden emission lines (e.g., [O I], [Fe II], [N II], and sometimes $\left[\mathrm{O}_{\mathrm{III}}\right]$ ) in addition to permitted ones, such as the Balmer and Fe II lines. From the very beginning, the phenomenon was recognized in stars with very different initial masses and at very different evolutionary stages.

Lamers et al. (1998) divided the original list of $\mathrm{B}[\mathrm{e}]$ stars into four subgroups according to the objects' evolutionary state: premain-sequence HAeB[e], symbiotic binaries (a cool giant and a white dwarf or a neutron star, symbB[e]), compact protoplanetary nebulae (PPNe)/planetary nebulae (cPNB[e]), and supergiants $(\mathrm{sgB}[\mathrm{e}])$. All the objects with unreliable or controversial information about their nature were separated into a fifth subgroup of unclassified (unclB $[\mathrm{e}]$ ) objects. These authors concluded that the $\mathrm{B}[\mathrm{e}]$ phenomenon is due to similar conditions in the circumstellar (CS) gaseous and dusty envelopes of these different types of objects.

The initial heterogeneity of the $\mathrm{B}[\mathrm{e}]$ star group has substantially hampered its study. As a result, unclB $[\mathrm{e}]$ objects that constituted $\sim 50 \%$ of the original group remained puzzling until recently. A few years ago, some of them were shown to have very specific IRAS colors that are unusual for hot stars with CS dust, such as HAeBe, Vega-type stars, Wolf-Rayet (W-R) stars, PPNe, and PNe (Sheikina et al. 2000). These colors (see Fig. 1, dashed box) reflect a steep flux decrease longward of $10-30 \mu \mathrm{m}$. Therefore, most of the CS dust near these objects has to be hot and close to the underlying star. The dust proximity to the star can be explained by either ongoing or recent dust formation.

In general, dust formation near hot stars requires special conditions, because the presence of significant amounts of energetic UV radiation destroys dust particles. Such conditions include strong stellar winds (responsible for high CS densities and thus shielding the dust grains from the stellar ionizing radiation), a high abundance of heavy elements (increasing the probability of dust formation), and clumping of the CS material (locally increasing the density). The conditions are natural near massive $\left(\geq 20 M_{\odot}\right)$ and luminous $\left(\geq 10^{5} L_{\odot}\right)$ objects, such as W-R stars, hot supergiants, and luminous blue variables (LBVs). Less massive and less luminous stars are not expected to have stellar winds strong enough to achieve CS densities that are sufficient for dust formation. Even rapidly rotating stars (e.g., Be stars) that lose mass at a higher rate are not capable of forming dust in their CS disks.

Further studies of unclB $[\mathrm{e}]$ showed that they are mostly lower luminosity $\left(2.5 \leq \log L / L_{\odot} \leq 4.5\right)$ objects (for a review, see Miroshnichenko et al. 2006). At the same time, Miroshnichenko et al. (2002) found more objects with the above-mentioned IRAS colors in the original list of $\mathrm{B}[\mathrm{e}]$ stars (Allen \& Swings 1976) and in a list of hot stars with extremely strong IR excesses (Dong \& $\mathrm{Hu}$ 1991). They also noted very strong emission-line spectra of these objects. It has been shown that a noticeable fraction of them are either recognized or suspected binary systems (Miroshnichenko et al. 2006). Thus mass transfer in binaries and formation of circumbinary disks can be an important mechanism responsible for accumulation of large amounts of CS gas and dust formation.
This group of unclB [e] with strong line emission and compact dusty envelopes has been known as $\mathrm{B}[\mathrm{e}]$ stars with warm dust or $\mathrm{B}[\mathrm{e}] \mathrm{WD}$. It initially included both $\mathrm{sgB}[\mathrm{e}]$ and lower luminosity unclB[e]. Finally, Miroshnichenko (2007) summarized previous studies of Galactic B[e] stars, added more candidates to the B[e]WD group from Allen \& Swings (1976), suggested separating sgB[e] from lower luminosity objects, called the latter FS CMa stars, and defined classification criteria for them.

The main distinguishing criteria for the FS CMa star group include the following:

1. Emission-line spectra containing hydrogen lines that are typically much stronger than those in the spectra of Be stars, $\mathrm{HAeBe}$, and normal supergiants; Fe II and [O I] lines; sometimes [Fe II] lines and weak [O III] lines.

2. A large IR excess that peaks at $10-30 \mu \mathrm{m}$ and sharply decreases longward, which is the locus in the dashed-line box in the IRAS color-color diagram of objects with all reliably measured fluxes at 12,25 , and $60 \mu \mathrm{m}$.

3. Location outside of star-forming regions.

4. When present, the secondary companion is $(a)$ if a normal star, typically fainter and cooler than the primary; or $(b)$ degenerate.

In a physical context, the underlying hot star (the primary companion in a binary) has an effective temperature $\left(T_{\text {eff }}\right)$ between $\sim 9000$ and $\sim 30,000 \mathrm{~K}$ (A2-09 type stars) and a luminosity range of $\log L / L_{\odot}=2.5-4.5$.

Miroshnichenko (2007) showed that these criteria separate FS CMa stars almost uniquely. The only confusion may occur with intermediate-mass post-AGB stars and with $\mathrm{sgB}[\mathrm{e}]$ due to uncertain luminosity. He pointed out that the FS CMa group was composed of the most prominent (the visually brightest and those with the strongest IR excesses) dust-forming B[e] stars and served as a basis for a new quest for more such objects. Indeed, we preliminarily reported nearly 30 new $\mathrm{B}[\mathrm{e}]$ star candidates in the IRAS Point Source Catalog ${ }^{2}$ (PSC; Miroshnichenko et al. Q2 2003a).

In this paper we describe this search for new $\mathrm{B}[\mathrm{e}]$ candidate objects and present new photometric criteria to separate postAGB stars from $B[\mathrm{e}]$ stars $(\S 2)$; present our initial spectroscopic and photometric observations of the newly found objects with the $\mathrm{B}[\mathrm{e}]$ phenomenon, as well as other objects with strong IR excesses and line emission $(\S 3)$; and discuss possible explanations for the observed features $(\S 4)$. As the newly found peculiar objects, especially those from the FS CMa group, show variations of both brightness and spectra, they require more observations to reveal their physics. We will continue accumulating data and publish detailed studies in future papers of this series.

\section{NEW SEARCH FOR GALACTIC B[e] STARS}

The initial selection of $\mathrm{B}[\mathrm{e}]$ objects (Allen \& Swings 1976) was based on spectroscopy (the presence of Fe II and forbidden emission lines) and near-IR photometry (the presence of a strong IR excess) with a threshold of $K \leq 8 \mathrm{mag}$. However, the compact location of FS CMa stars in the IRAS color-color diagram in combination with a hot-star optical continuum (although usually

${ }^{2}$ IRAS Catalog of Point Sources (ver. 2.0, 1986; Pasadena: IPAC). 


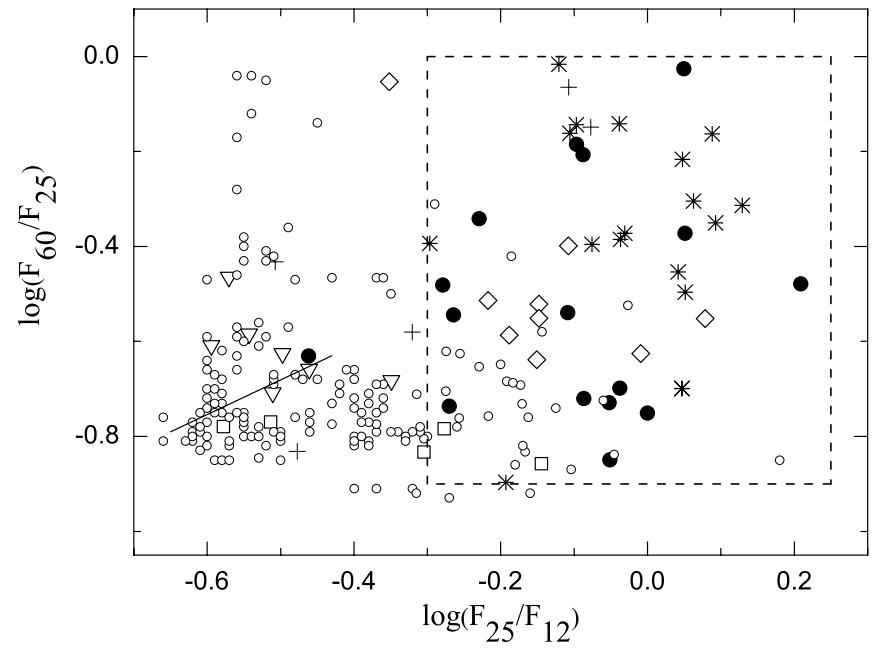

FIG. 1.—IRAS color-color diagram for various dusty objects: previously known FS CMa stars, filled circles; VV Cep binaries, open squares; carbon stars, downward triangles; M-type stars including Miras, small open circles; Wolf-Rayet stars, plus signs; $\mathrm{RV}$ Tau stars, open diamonds; and new candidates for stars with the $\mathrm{B}[\mathrm{e}]$ phenomenon, asterisks. The solid line represents interstellar extinction from the locus of the photospheric colors to $A_{V}=20 \mathrm{mag}$. The most probable location of stars with the $\mathrm{B}[\mathrm{e}]$ phenomenon is the dashed box, which also contains some M-type, W-R, and RV Tau stars.

reddened) and certain near-IR color-indices due to the presence of CS dust permits a search that is based on photometric criteria. Moreover, the optical brightness of FS CMa stars ( $V \leq 14 \mathrm{mag}$ ), their IR fluxes of $\sim 3-150 \mathrm{Jy}$ at $12-25 \mu \mathrm{m}$, and their distances from the Sun $(0.3-3.5 \mathrm{kpc})$ along with the sparse spectroscopic data suggest that more such objects could still have been detected by $I R A S$.

The main problem with IRAS PSC searches is the catalog's low positional accuracy $\left(10^{\prime \prime}-30^{\prime \prime}\right)$. Fortunately, FS CMa stars have strong near-IR excesses that allow us to use the 2MASS catalog (Cutri et al. 2003) to refine the IRAS positions. This is possible because $I R A S$ sources are typically associated with the brightest 2MASS sources in the IRAS positional error box, except for extremely crowded areas. In addition, we also need an optical catalog that contains both accurate positions and some kind of multicolor photometry. The USNO-B1.0 catalog (Monet et al. 2003) has the required properties. Both catalogs (USNO-B1.0 and 2MASS) have an astrometric accuracy of $1^{\prime \prime}-2^{\prime \prime}$, and their positions are usually consistent within a fraction of an arcsecond, therefore assuring unique identifications of the IRAS sources.

In order to find new FS CMa stars, we selected IRAS sources with colors within the dashed box (Fig. 1), searched for the brightest 2MASS sources within the IRAS positional error boxes (typically within $30^{\prime \prime}$ ) and with $H-K$ colors that are redder than a cool star continuum (typically $H-K \geq 0.5 \mathrm{mag}$ ), and selected those associated with not very red USNO-B1.0 objects. The latter procedure is a bit ambiguous, because FS CMa stars usually exhibit significant optical reddening, so that their optical color indices may be confused with those of unreddened cool stars. However, most unreddened or mildly reddened cool stars, which have IRAS colors similar to those of FS CMa stars, are well separated from the latter by near-IR color indices.

In our search, we selected an initial sample of nearly 4500 IRAS sources with good-quality flux measurements in all three photometric bands $(12,25$, and $60 \mu \mathrm{m})$. This sample was reduced to about 100 candidates after applying the above constraints on the 2MASS and USNO-B1.0 data. Our procedure found all previously known FS CMa stars (Miroshnichenko 2007). On the

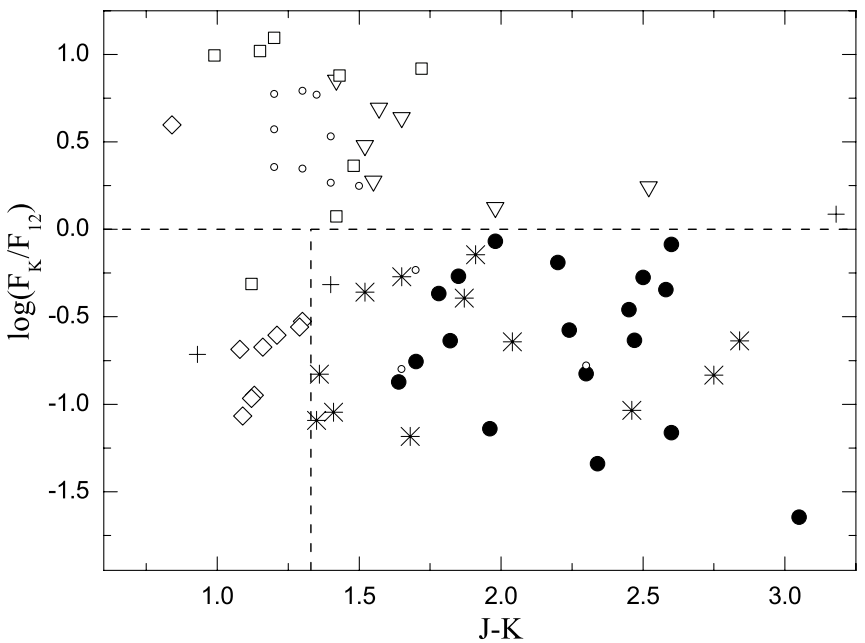

FIG. 2.-Color-color diagram for selecting FS CMa stars. The symbols represent the same groups of object as in Fig. 1. Most of the M-type stars seen in Fig. 1 are out of the range here. They have larger $F_{K} / F_{12}$ flux ratios. The dashed lines show empirical separation between cool and hot stars with CS dust. The stars in the lower right corner are almost exclusively known FS CMa stars and new candidates reported here.

other hand, our candidate list also turned out to contain known RV Tau stars, which are low-luminosity post-AGB objects (e.g., Lloyd Evans 1985).

Analyzing different color-color diagrams, we developed photometric criteria to separate cool and hot dusty stars. It turned out that cool stars with even moderately thick dusty envelopes have a $K$-band $(2.2 \mu \mathrm{m})$ flux that exceeds the IRAS $12 \mu \mathrm{m}$ band flux $\left(\log F_{K} / F_{12} \geq 0\right.$; Fig. 2). Cool stars with the thickest CS dust have negative $\log F_{K} / F_{12}$, but they are much redder $(V-K \geq$ $8 \mathrm{mag}$ ) than FS CMa stars ( $V-K \leq 7 \mathrm{mag})$. Also, RV Tau stars have smaller near-IR color-indices than known FS CMa stars (e.g., $J-K \leq 1.3 \mathrm{mag}$ ).

Using the $\log F_{K} / F_{12}$ versus $J-K$ diagram, we excluded the RV Tau stars from the sample. This left us with about 30 candidates for objects with the $\mathrm{B}[\mathrm{e}]$ phenomenon. We should stress here that since the USNO-B1.0 catalog is based on photographic photometry, it cannot be considered to have a good absolute flux calibration, so that we are unable to constrain the objects' spectral energy distributions (SEDs) in the optical region. Therefore, spectroscopy is the ultimate tool to select the best candidates for inclusion in the FS CMa group. Our photometric and spectroscopic observations, reported in $\S 3$, allowed us to expand the list of FS CMa stars.

\section{OBSERVATIONS}

The spectroscopic observations of the new $\mathrm{B}[\mathrm{e}]$ star candidates were obtained at several telescopes in 2003-2006. We started from low-resolution spectroscopy at small telescopes and highresolution spectroscopy in small wavelength ranges, typically near $\mathrm{H} \alpha$ and [ $\left.\mathrm{O}_{\mathrm{I}}\right]$ lines. Information about the telescopes and spectrographs we used is presented in Table 1 . These data allowed us to select genuine objects with the $\mathrm{B}[\mathrm{e}]$ phenomenon. More recently we acquired high-resolution echelle spectra to begin their more comprehensive study. Some of our data sets are relatively (Dark Sky Observatory data) or absolutely (Lick Observatory data) flux calibrated. This allowed us to check and confirm the association of the IRAS sources with the optical and 2MASS counterparts. In the course of our observations, we also found a few objects with no clear signature of the $\mathrm{B}[\mathrm{e}]$ phenomenon, but with an unusually large IR excess and/or spectral line emission. 
TABLE 1

Telescopes and Instruments Used for the Spectroscopic Observations

\begin{tabular}{|c|c|c|c|c|}
\hline $\begin{array}{l}\text { Telescope } \\
\text { (1) }\end{array}$ & $\begin{array}{l}\text { Spectrograph } \\
\text { (2) }\end{array}$ & $\begin{array}{c}\text { Spectral Region }(\AA) \\
\text { (3) }\end{array}$ & $\begin{array}{l}R \\
(4)\end{array}$ & $\begin{array}{l}\text { Reference } \\
\text { (5) }\end{array}$ \\
\hline 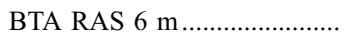 & NES & $5000-6700$ & 60000 & 1 \\
\hline 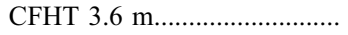 & ESPaDOnS & $3600-10480$ & 70000 & 2,3 \\
\hline 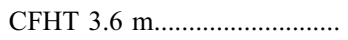 & Gecko & $6500-6620$ & 100000 & 4 \\
\hline 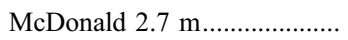 & $\operatorname{cs} 2$ & $3600-10500$ & 60000 & 5 \\
\hline 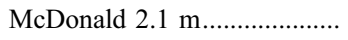 & Sandiford & $5400-6700$ & 60000 & 6 \\
\hline 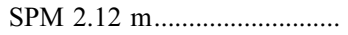 & REOSC & $3850-6850$ & 20000 & 7 \\
\hline Loiano $1.5 \mathrm{~m}$ & BFOSC & $3800-8000$ & 5000 & 8 \\
\hline DSO $0.8 \mathrm{~m}$ & Gray-Miller & $3800-5600$ & 1300 & 9 \\
\hline Lick 3 m ....... & NIRI & $4600-24500$ & 700 & 10 \\
\hline
\end{tabular}

Notes.-Col. (1): The telescopes (BTA RAS: the $6 \mathrm{~m}$ telescopes of the Russian Academy of Sciences, Russia; CFHT: the 3.6 m Canada-France-Hawaii Telescope; SPM: San Pedro Martir Observatory, Mexico; DSO: Dark Sky Observatory, Boone, NC; Loiano: Loiano Observatory, Bologna, Italy). Col. (2): The names of the spectrographs. Col. (3): Spectral region observed. Col. (4): Spectral resolving power $R$. Col. (5): Reference to the spectrograph description in col. (5).

References.- (1) Panchuk et al. 1999; (2) Donati et al. 1997; (3) Manset \& Donati 2003; (4) Baudrand \& Vitry 2000; (5) Tull et al. 1995; (6) McCarthy et al. 1993; (7) Levine \& Chakrabarty 1995; (8) Gualandi \& Merighi 2001; (9) Gray et al. 2003; (10) Rudy et al. 1999.

The photometric observations were obtained at the following observatories. At the Tien-Shan Observatory (Kazakhstan) and the Majdanak Observatory (Uzbekistan) we used single-element pulse-counting photometers with a set of broadband UBVR Johnson filters. At the Lizard Hollow Observatory (Tucson, AZ) and at the Loiano Observatory (Bologna, Italy) we used CCD photometers with a set of $U B V\left(R I_{c}\right)$ filters. A few observations were obtained for most of the objects. As they all exhibit brightness variations, we only report average photometric data here.

Observational parameters of the selected objects with the $\mathrm{B}[\mathrm{e}]$ phenomenon are listed in Table 2 and those of the other interesting objects are listed in Table 3 . The results of our photometric observations are presented in Table 4.

\subsection{New Objects with the $\mathrm{B}[\mathrm{e}]$ Phenomenon}

All the objects from Table 2 show [O I] forbidden emission lines in their spectra in addition to the strong Balmer emission lines. This, in combination with their strong IR excess (see Fig. 20) and IRAS color indices, strongly favors their classification as dustforming $\mathrm{B}[\mathrm{e}]$ stars and specifically as FS CMa stars. In this section we discuss the objects' properties separately.

\subsubsection{FBS 0022-021}

FBS 0022-021, a $V=15$ mag object, appeared in the First Byurakan Survey for active Galactic nuclei (Markarian et al. 1989). It was suspected to be a U Gem type cataclysmic variable (CV; Downes et al. 2001), but has not been confirmed (Kopylov et al. 1988). Its strong IR excess ( $V-K=4 \mathrm{mag}$; Hoard et al. 2002 ) is typical of FS CMa stars and should be due to dust, since the optical reddening is weak $(B-V=0.1 \mathrm{mag})$. In contrast, CVs do not show signs of dust. Its emission-line spectrum (mostly $\mathrm{H}$, Fe II, [Fe II], [O I] ) is similar to that of $\eta$ Carinae (Zharikov et al. 2004) and the strongest among the FS CMa stars. Its Galactic latitude of $-64^{\circ}$ makes it unique among the Population I dustforming $\mathrm{B}[\mathrm{e}]$ stars and (along with its IR excess) among other highlatitude B-type stars. Its radial velocity of $-50 \mathrm{~km} \mathrm{~s}^{-1}$ indicates that it belongs to our Galaxy. The photometric distance is $7.5 \mathrm{kpc}$ (if a dwarf) or $\geq 20 \mathrm{kpc}$ (if a supergiant) and makes it the most distant Galactic object with the $\mathrm{B}[\mathrm{e}]$ phenomenon, unless it is intrinsically underluminous or attenuated by gray extinction.

Our CFHT spectrum has an insufficient signal-to-noise ratio to reveal faint features near continuum (Fig. 3). In particular, no traces of $\mathrm{He}$ I and $\mathrm{He}$ II lines were detected. This may indicate a

TABLE 2

New Stars with the B[e] Phenomenon

\begin{tabular}{|c|c|c|c|c|c|c|c|c|c|c|c|c|}
\hline $\begin{array}{l}I R A S \text { ID } \\
\quad(1)\end{array}$ & $\begin{array}{l}\text { Name } \\
(2)\end{array}$ & $\begin{array}{l}\text { R.A. } \\
\text { (2) }\end{array}$ & $\begin{array}{l}\text { Decl. } \\
\text { (4) }\end{array}$ & $\begin{array}{c}V \\
(5)\end{array}$ & $\begin{array}{l}B-V \\
(6)\end{array}$ & $\begin{array}{c}J-K \\
(7)\end{array}$ & $\begin{array}{c}K \\
(8)\end{array}$ & $\begin{array}{l}F_{12} \\
(9)\end{array}$ & $\begin{array}{l}F_{25} \\
(10)\end{array}$ & $\begin{array}{l}F_{60} \\
(11)\end{array}$ & $\begin{array}{c}\text { Spectral Type } \\
\text { (12) }\end{array}$ & $\begin{array}{c}\text { Reference } \\
\text { (13) }\end{array}$ \\
\hline (none). & FBS $0022-021$ & 002452.6 & -015335.3 & 15.0 & 0.08 & 2.37 & 11.10 & $\cdots$ & & $\ldots$ & B5 & 1 \\
\hline $03421+2935 \ldots \ldots \ldots \ldots$ & MWC 728 & 034514.7 & +294503.2 & 9.8 & 0.2 & 1.65 & 6.92 & 2.10 & 2.60 & 1.16 & $\mathrm{~B} 6 / 7+\mathrm{K}$ & 3 \\
\hline $06071+2925 \ldots \ldots \ldots \ldots$ & PDS 211 & 061017.4 & +292516.7 & 13.7 & 0.85 & 1.68 & 9.81 & 1.20 & 1.47 & 1.01 & $\mathrm{~B} / \mathrm{A}$ & 4 \\
\hline $07080+0605 \ldots \ldots \ldots \ldots$ & & 071043.9 & +060008.0 & 12.2 & 0.15 & 2.84 & 7.00 & 4.54 & 3.63 & 2.61 & A0 & 2 \\
\hline 08307-3748 ……..... & FX Vel & 083235.8 & -375901.5 & 9.7 & & 1.36 & 7.81 & 3.34 & 3.11 & 1.32 & $A+K$ & 6 \\
\hline $17449+2320 \ldots \ldots \ldots \ldots$ & $\mathrm{BD}+233183$ & 174703.3 & +231945.4 & 10.0 & 0.06 & 1.87 & 7.21 & 2.13 & 1.79 & 0.72 & A0 & 2 \\
\hline
\end{tabular}

Notes.-The object's IRAS ID is listed in col. (1); the optical counterpart's ID in col. (2); equatorial coordinates from USNO-B1.0 and 2MASS in cols. (3) and (4); visual magnitude and $B-V$ color index in cols. (5) and (6); the 2MASS $J K$ data in cols. (7) and (8); the IRAS fluxes in Jy in cols. (9)-(11); spectral type estimate from our spectra in col. (12); and references for the optical photometry in col (13). Units of right ascension are hours, minutes, and seconds, and units of declination are degrees, arcminutes, and arcseconds (J2000.0).

References.-(1) Zharikov et al. 2004; (2) this work; (3) Tycho-2 catalog (Hog et al. 2000); (4) Vieira et al. 2003; (5) Orsatti 1992; (6) Eggen 1978. 
TABLE 3

Other Objects with Line Emission and Strong IR Excesses

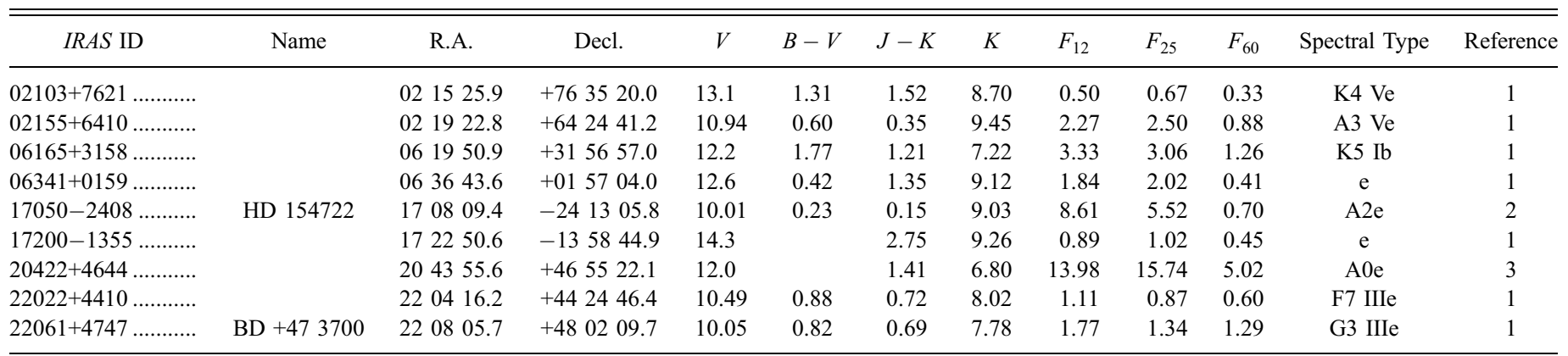

NoteS.-The object's IRAS ID is listed in col. (1); the optical counterpart's ID in col. (2); equatorial coordinates from USNO-B1.0 and 2MASS in cols. (3) and (4); visual magnitude and $B-V$ color index in cols. (5) and (6); the 2MASS $J K$ data in cols. (7) and (8); the IRAS fluxes in Jy in cols. (9)-(11); spectral type estimate from our spectra in col. (12); and references for the optical photometry in col (13). Units of right ascension are hours, minutes, and seconds, and units of declination are degrees, arcminutes, and arcseconds (J2000.0)

References.-(1) This work; (2) Tycho-2 catalog (Hog et al. 2000); (3) USNO-B1.0 catalog (Monet et al. 2003).

relatively low $T_{\text {eff }}$ of the underlying star (no earlier than a mid-B spectral type). It is unclear how much veiling distorts the spectrum and what the intrinsic photospheric brightness and stellar luminosity are. The object's SED is shown in Zharikov et al. (2004).

\subsubsection{IRAS $00470+6429$}

This object was detected in a number of emission-line star surveys, such as Gonzalez \& Gonzalez (1954), Dolidze \& Ponomareva (1975), Coyne \& MacConnel (1983), and Kohoutek \& Wehmeyer (1999). Meixner et al. (1999) imaged it at $\lambda=10.6 \mu \mathrm{m}$ as a PPN candidate, but found it unresolved. Kelly \& Hrivnak (2005) took a near-IR spectrum near the $\lambda=2.2 \mu \mathrm{m} \mathrm{Br} \gamma$ line and found this line to be strongly in emission, but they did not detect molecular $\mathrm{H}_{2}$ features and have not confirmed the PPN status.

Our spectra show very strong Balmer emission lines with $\mathrm{P}$ Cyg profiles (Fig. 4), numerous Fe II emission lines, and some [ $\mathrm{Fe}$ II] emission lines. We detected a few weak $\mathrm{He}$ I lines (e.g., 5876 and $6678 \AA$ ) in emission, indicating that the underlying star has roughly a B2/B3 spectral type. This estimate is consistent with our $U B V R$ data (see Table 4). It is definitely not an A-type star, as Kelly \& Hrivnak (2005) suggested. The P Cyg profiles of the Balmer lines and the strongest Fe II lines indicate that the CS gas is distributed either nearly spherically or in a torus with a relatively large opening angle. This is rare, but not unusual in FS CMa

TABLE 4

Our Photometric Data for the Objects

\begin{tabular}{ccccccc}
\hline \hline $\begin{array}{c}\text { IRAS ID } \\
(1)\end{array}$ & $\begin{array}{c}V \\
(2)\end{array}$ & $\begin{array}{c}U-B \\
(3)\end{array}$ & $\begin{array}{c}B-V \\
(4)\end{array}$ & $\begin{array}{c}V-R \\
(5)\end{array}$ & $\begin{array}{c}V-I \\
(6)\end{array}$ & $\begin{array}{c}\text { Source } \\
(7)\end{array}$ \\
\hline $00470+6429 \ldots \ldots \ldots$. & 12.09 & 0.19 & 0.97 & 1.08 & $\ldots$ & Majdanak \\
$02155+6410 \ldots \ldots \ldots$. & 10.94 & -0.02 & 0.60 & 0.50 & $\ldots$ & TSAO \\
$06165+3158 \ldots \ldots \ldots$. & 12.17 & $\ldots$ & 1.81 & 1.8 & $\ldots$ & TSAO \\
$06341+0159 \ldots \ldots \ldots$. & 12.15 & $\ldots$ & 0.26 & 0.56 & $\ldots$ & TSAO \\
$07080+0605 \ldots \ldots \ldots$. & 12.15 & -0.01 & 0.16 & 0.25 & 0.46 & LHO, Loiano \\
$17449+2320 \ldots \ldots \ldots$. & 10.00 & -0.01 & 0.06 & 0.16 & $\ldots$ & Majdanak \\
$22022+4410 \ldots \ldots \ldots$. & 10.49 & 0.10 & 0.88 & 0.72 & $\ldots$ & TSAO \\
$22061+4747 \ldots \ldots \ldots$. & 10.05 & 0.05 & 0.82 & 0.67 & $\ldots$ & TSAO \\
\hline
\end{tabular}

Notes.-The IRAS ID is listed in col. (1); the average photometric data in cols. (2)-(6); and the observatory, at which the data were taken, in col. (7). The data from the Tien-Shan (TSAO) and Majdanak Observatories are obtained in the Johnson $R$ filter, while those from the Lizard Hollow Observatory (LHO) and Loiano Observatory are obtained in the Cousins $R I$ filters. Typical measurement uncertainties are $0.02-0.04 \mathrm{mag}$. The $V-R$ color-index of IRAS $06165+3158$ has an uncertainty of $0.1 \mathrm{mag}$. stars (see, e.g., Miroshnichenko et al. 2000) and may indicate a certain phase of the $\mathrm{B}[\mathrm{e}]$ phenomenon development.

IRAS $00470+6429$ is located in the line of sight of the association Cas OB7, whose distance is $2 \mathrm{kpc}$ (Cazzolato \& Pineault 2003). However, the interstellar components of its $\mathrm{Na}$ I lines have a simpler structure (one component) than those of the association members. Therefore, IRAS $00470+6429$ is a closer star with a nearly main-sequence luminosity.

\subsubsection{IRAS $03421+2935$}

IRAS $03421+2935$, a relatively bright object $(V \sim 10 \mathrm{mag}$ ), was first detected in the Mount Wilson survey for Galactic earlytype emission-line sources (MWC 728; Merrill \& Burwell 1949). The optical counterpart is also known as HD 281192. No optical spectroscopy or photometry has been published for the star. te Lintel Hekkert et al. (1991) and Chengalur et al. (1993) observed it in the $\mathrm{OH}$ maser lines at $1612 / 1667 \mathrm{MHz}$ because its IRAS colors are similar to those of $\mathrm{OH} / \mathrm{IR}$ stars, but did not detect any line emission. Our spectra show double-peaked Balmer and [O I] lines in emission (Fig. 5). The He I 4471/Mg II 4481 line equivalent width ratio, which equals to 1.7 , suggests a $\mathrm{B} 6 / \mathrm{B} 7$ spectral type (according to a calibration of Kopylov et al. 1989), consistent with
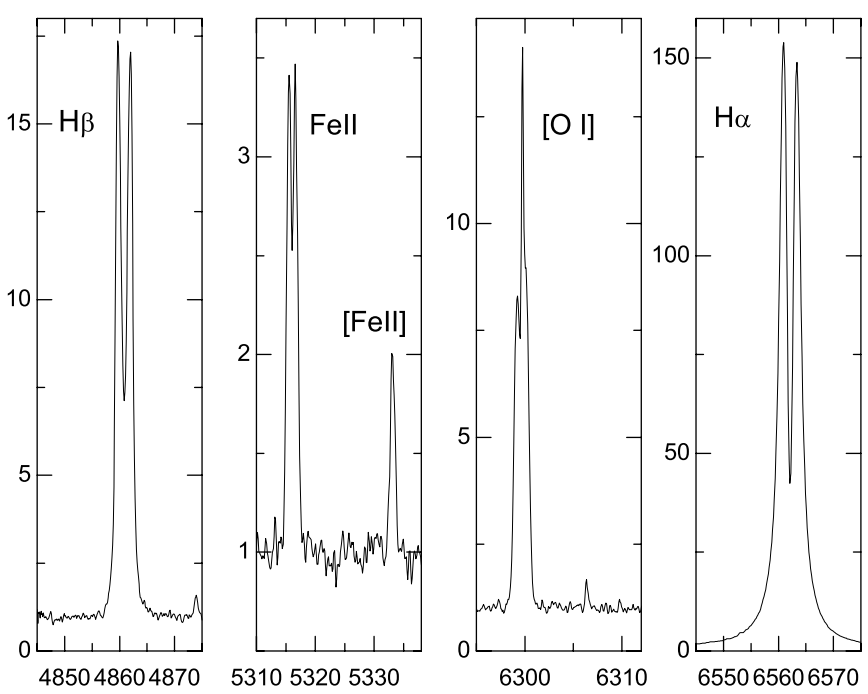

Fig. 3.-Portions of the optical high-resolution spectrum of FBS 0022-021. The spectrum was obtained at the $3.6 \mathrm{~m} \mathrm{CFHT} \mathrm{in} 2006$ January. Intensities are normalized to the underlying continuum, and wavelengths are given in $\AA$. 


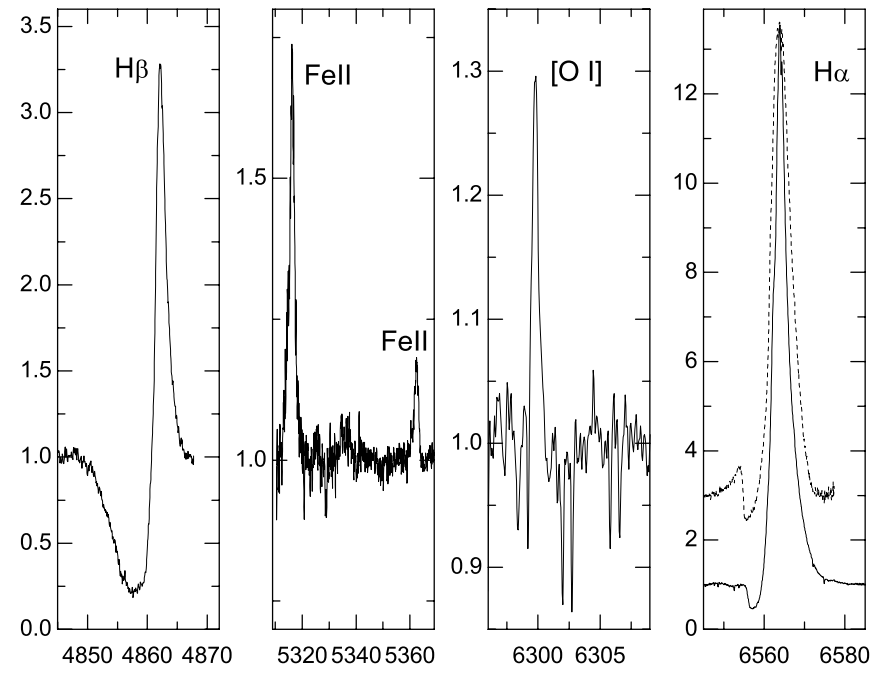

FIG. 4.-Portions of the optical high-resolution spectrum of IRAS $00470+6429$. The solid line represents the spectrum obtained at the $2.7 \mathrm{~m}$ Harlan J. Smith Telescope of the McDonald Observatory (2005 December), and the dashed line shows the $\mathrm{H} \alpha$ line profile from the spectrum obtained at the $6 \mathrm{~m}$ telescope of the Russian Academy of Sciences (2003 August). Intensities and wavelengths are in the same units as in Fig. 3.

the Tycho-2 photometric data (Hog et al. 2000). The absence of Fe II emission lines indicates an even lower $T_{\text {eff, }}$ although the large equivalent width $(\mathrm{EW})$ of $\mathrm{H} \alpha(\sim 50 \AA)$ and very strong [O I ] lines are unusual for such a cool star. We have also detected the weak Li I $6708 \AA$ and Ca I $6717 \AA$ lines that are indicative of a late-type companion (Fig. 6).

\subsubsection{IRAS $06071+2925$}

The optical counterpart of IRAS $06071+2925$ was identified in the course of the Pico dos Dios survey for young stars (PDS 211; Vieira et al. 2003). These authors obtained one UBVRI photometric observation ( $B V$ data shown in Table 2$)$ and a medium-resolution spectrum that showed a strong double-peaked $\mathrm{H} \alpha$ line and [O I] 6300, $6363 \AA$ line emission. They classified the object as an A-type star. Our spectra (Fig. 7) do not contradict the spectral type, although they are not good enough to refine it and suggest a range from late-B to early-A. They also indicate a low luminosity (as



FIG. 5.- Portions of the optical high-resolution spectrum of IRAS 03421+ 2935. The spectrum was obtained at the $3.6 \mathrm{~m}$ CFHT in 2004 December. Intensities and wavelengths are in the same units as in Fig. 3.

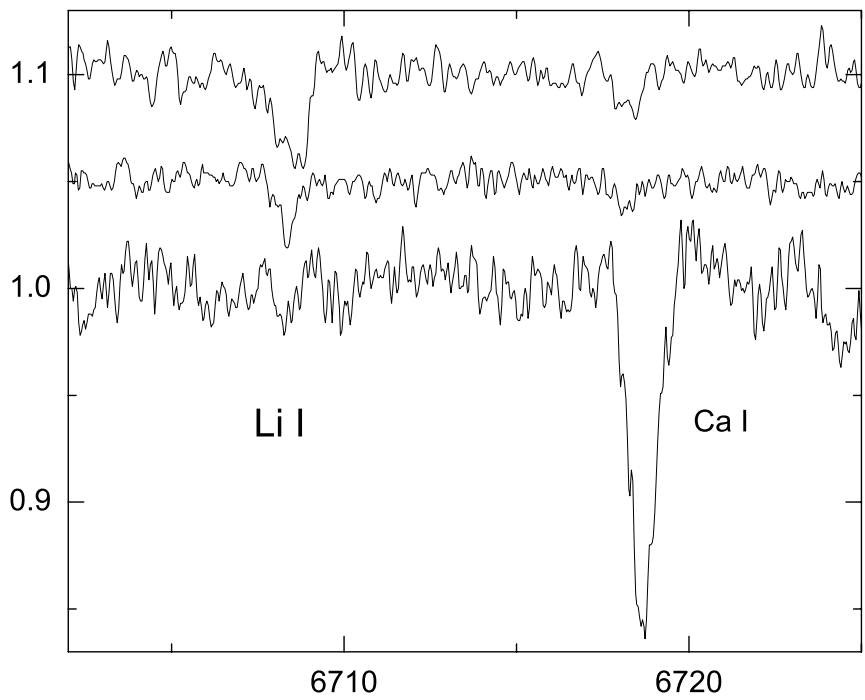

FIG. 6.- Portions of the optical high-resolution spectra of the objects with evidence for a late-type companion. The data were obtained at the $3.6 \mathrm{~m} \mathrm{CFHT}$ (2004 December). From top to bottom, shifted by 0.05 of the continuum intensity: IRAS 03421+2935, IRAS 08307-3748, and IRAS 07455-3143. Intensities and wavelengths are in the same units as in Fig. 3.

the $\mathrm{Si}$ II lines are weak). The Fe II emission is weak, but the He I lines (e.g., 5876, 6678, 7065, and $10830 \AA$ ) are present in emission. Such a combination is unusual in low-temperature objects with the $\mathrm{B}[\mathrm{e}]$ phenomenon and may indicate the presence of a companion that is responsible for the He I excitation. We also detected strong variations of the $\mathrm{H} \alpha$ line profile.

\subsubsection{IRAS $07080+0605$}

The optical counterpart of IRAS $07080+0605$ was detected by Kohoutek \& Wehmeyer (1999) in their survey for H $\alpha$-emitting stars. No other data have been reported in the literature. Our spectra suggest that the underlying star has an A spectral type and a low luminosity (broad wings of the $\mathrm{H} \beta$ and higher members of

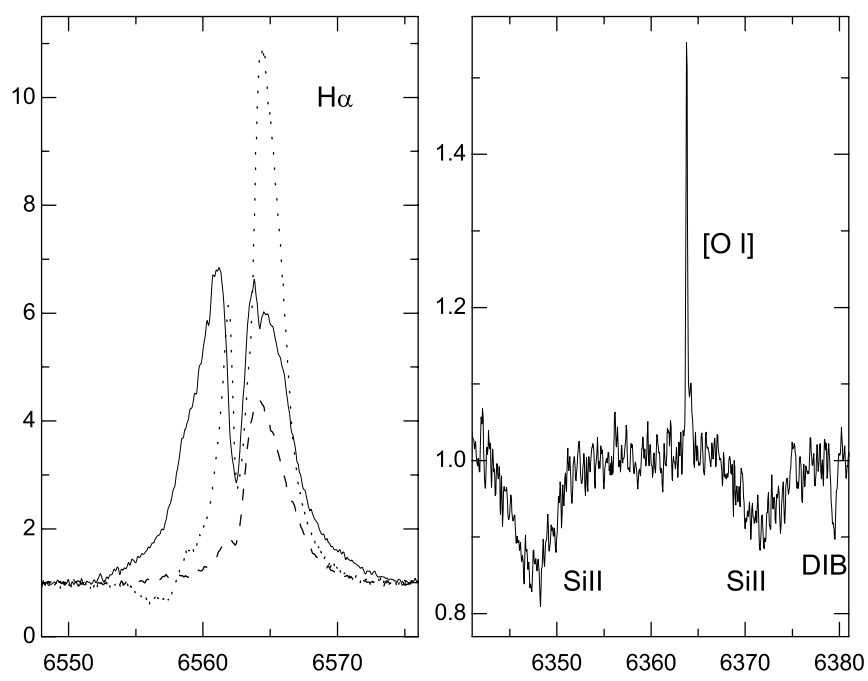

FIG. 7.- Portions of the optical high-resolution spectrum of IRAS 06071+2925. Left panel: The dashed line represents $\mathrm{H} \alpha$ line profile from the spectrum obtained at the $2.7 \mathrm{~m}$ Harlan J. Smith Telescope of the McDonald Observatory (2005 December), the solid line represents the spectrum obtained at the $3.6 \mathrm{~m}$ CFHT (2006 February), and the dotted line shows the CFHT spectrum from 2004 February. Right panel: The $\mathrm{Si}$ II 6347 and $6371 \AA$ lines, the [O I] $6363 \AA$ line, and the diffuse interstellar band (DIB) at $6379 \AA$ from the 2005 December McDonald spectrum. Intensities and wavelengths are in the same units as in Fig. 3. 

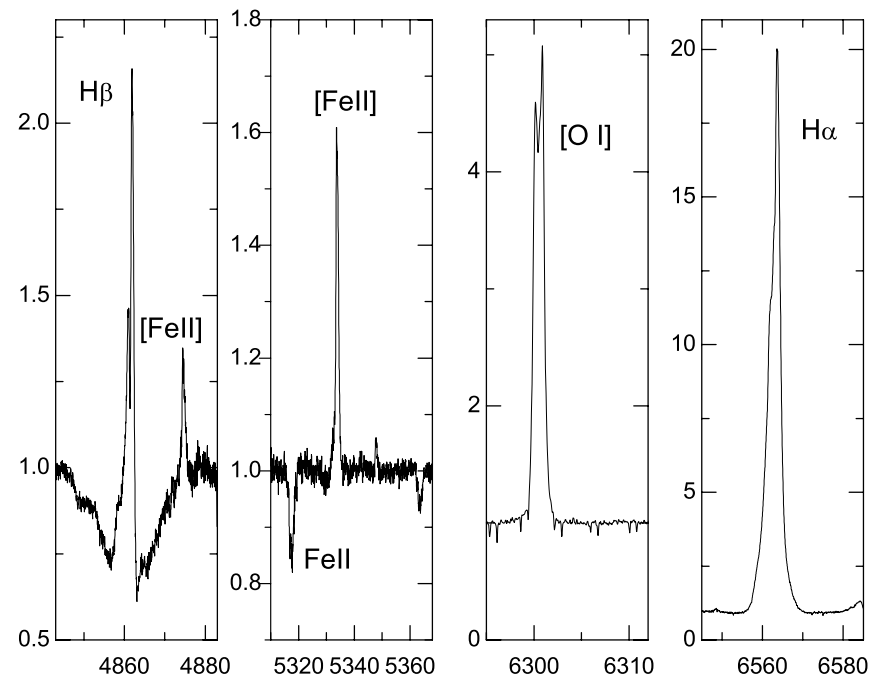

FIG. 8.-Portions of the optical high-resolution spectrum of IRAS $07080+0605$. The spectrum was obtained at the $3.6 \mathrm{~m}$ CFHT in 2004 December. Intensities and wavelengths are in the same units as in Fig. 3.

the Balmer series line, Fig. 8). This is consistent with our photometry (Table 2) and with the $\mathrm{He}_{\mathrm{I}} 4471 / \mathrm{Mg}_{\text {II }} 4481$ line ratio (4.2). As in the case of IRAS $03421+2935$, the $\mathrm{H} \alpha$ line $(\mathrm{EW}=68 \AA)$ is extremely strong for the spectral type. Its profile is most likely double-peaked, as are those of $\mathrm{H} \beta$ and [O I] lines, but the peak separation is small $\left(\sim 50 \mathrm{~km} \mathrm{~s}^{-1}\right)$ and hardly detectable even at our spectral resolution $(R=60,000)$.

Surprisingly, the object has one of the strongest IR excesses among the objects with the $\mathrm{B}[\mathrm{e}]$ phenomenon (see Fig. 20). Along with the remarkable $\mathrm{H} \alpha$ emission, this strongly favors the accumulation of the CS material rather than the regular stellar wind. The latter should be unexpectedly powerful for such a low $T_{\text {eff }}$ and luminosity object. A natural explanation for this behavior can be rapid mass transfer in a binary system. However, we see no direct evidence for the secondary companion in our spectra.

\subsubsection{IRAS 07377-2523}

IRAS $07377-2523$ was selected as a post-AGB candidate by Parthasarathy et al. (2000), who took a blue spectrum of its optical counterpart and found it to be a late B-type star (B8 III-IVe) with the only emission in the $\mathrm{H} \beta$ line and no emission in the $\mathrm{H} \gamma-\mathrm{H} 8$ lines. These authors have not classified the object as a post-AGB star. Our spectrum shows double-peaked Balmer and Fe II emission-line profiles with a strong central depression (Fig. 9). This indicates an edge-on orientation of the gaseous CS disk. The $\mathrm{He}_{\text {I }} 4471 / \mathrm{Mg}$ II 4481 line ratio $(\sim 1)$ is consistent with a B8/A0 spectral type. The $\mathrm{H} \alpha$ strength ( $\mathrm{EW} \sim 65 \AA$ ) is comparable to that of IRAS $07080+0605$.

\subsubsection{IRAS $07455-3143$}

IRAS 07455-3143 was detected as an $\mathrm{H} \alpha$ emitter in the Mount Wilson survey (AS 174; Merrill \& Burwell 1950) and in a survey for southern emission-line stars by Henize (1976). It was included in the Luminous Stars for the Southern Milky Way survey (LS 782; Stephenson \& Sanduleak 1971) and later in a catalog of luminous stars (ALS 782; Reed 1998). UBV photometry by Orsatti (1992) shows that it is a reddened early B-type star $(U-B=-0.12, B-V=0.92)$.

Our spectrum (Fig. 10) is similar to that of IRAS 073772523, with double-peaked Balmer and Fe II line profiles. However, IRAS $07455-3143$ exhibits [ $\mathrm{Fe}$ II] emission lines instead
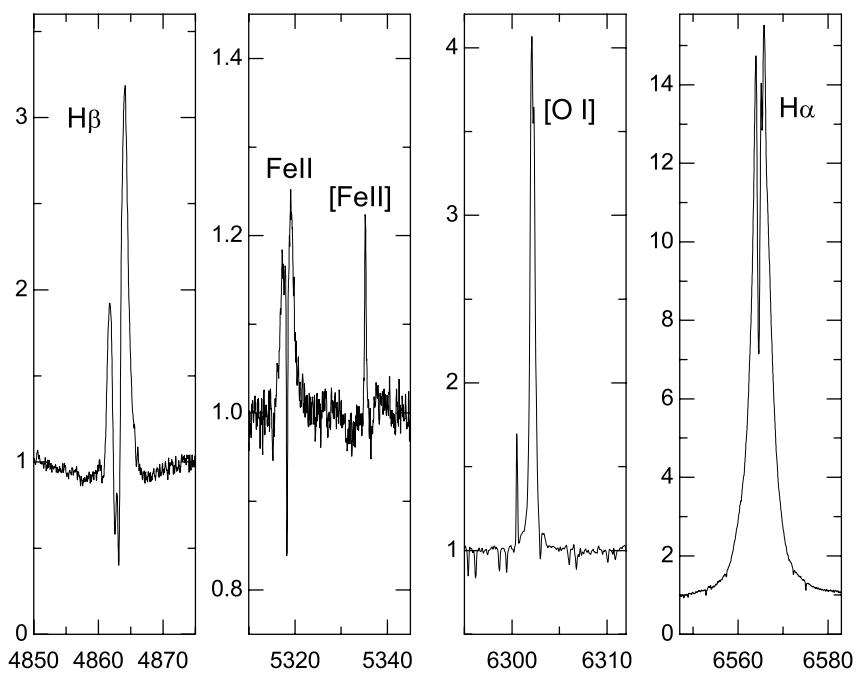

FIG. 9.- Portions of the optical high-resolution spectrum of IRAS 07377-2523. The spectrum was obtained at the $3.6 \mathrm{~m}$ CFHT in 2004 December. The telluric and CS components of the [O I] $6300 \AA$ line are clearly separated. Intensities and wavelengths are in the same units as in Fig. 3.

of the $\left[\mathrm{O}_{\mathrm{I}}\right]$ lines, which are very narrow and may have telluric origin. The He I $4471 / \mathrm{Mg}$ II 4481 line ratio $(\sim 1.1)$ indicates a B7/ B8 spectral type, but the He I line may be affected by the CS emission. IRAS $07455-3143$ shows evidence for a late-type companion through the presence of the lines of Li I $6708 \AA$ and Ca I $6717 \AA ̊$ (see Fig. 6).

\subsubsection{IRAS 08307-3748}

IRAS $08307-3748$ is another object from the Mount Wilson survey (AS 202; Merrill \& Burwell 1950). Strohmeier et al. (1968) found strong optical variability from a photographic patrol (amplitude $1.20 \mathrm{mag}$ ), which earned the object its variable star name FX Vel (Kukarkin et al. 1972) and classification as an eclipsing binary. Eggen (1978) did not detect the reported variations in his Strömgren photometry and put in doubt the object's eclipsing nature. Nevertheless, Malkov et al. (2006) included FX Vel in their new catalog of eclipsing binaries with the depths of the primary $(1.0 \mathrm{mag})$ and secondary $(0.8 \mathrm{mag})$ eclipses and a period of 1.052 days mentioned by Eggen (1978).

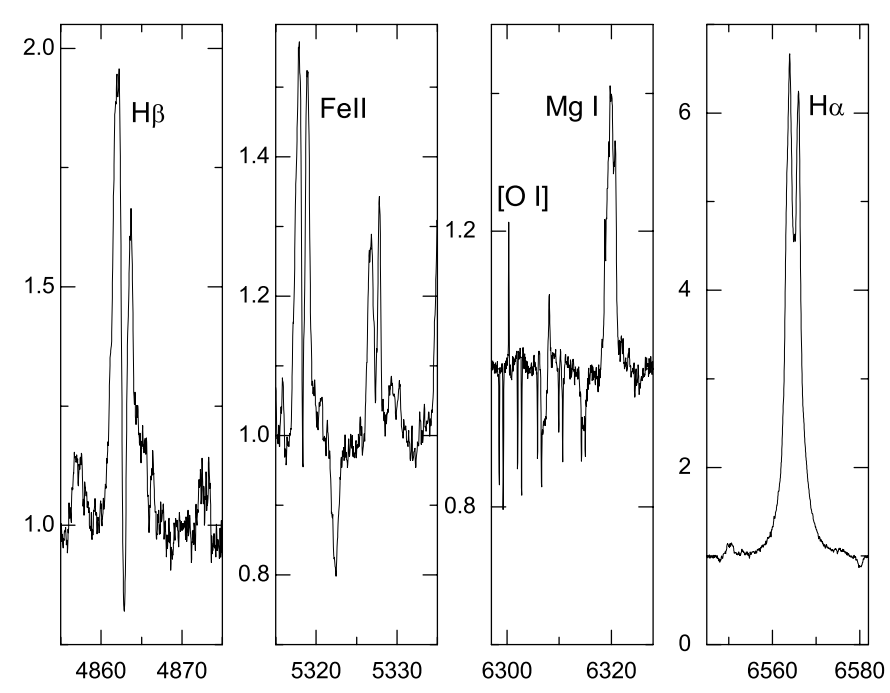

FIG. 10.-Portions of the optical high-resolution spectrum of IRAS 07455-3143. The spectrum was obtained at the $3.6 \mathrm{~m}$ CFHT in 2004 December. Intensities and wavelengths are in the same units as in Fig. 3. 


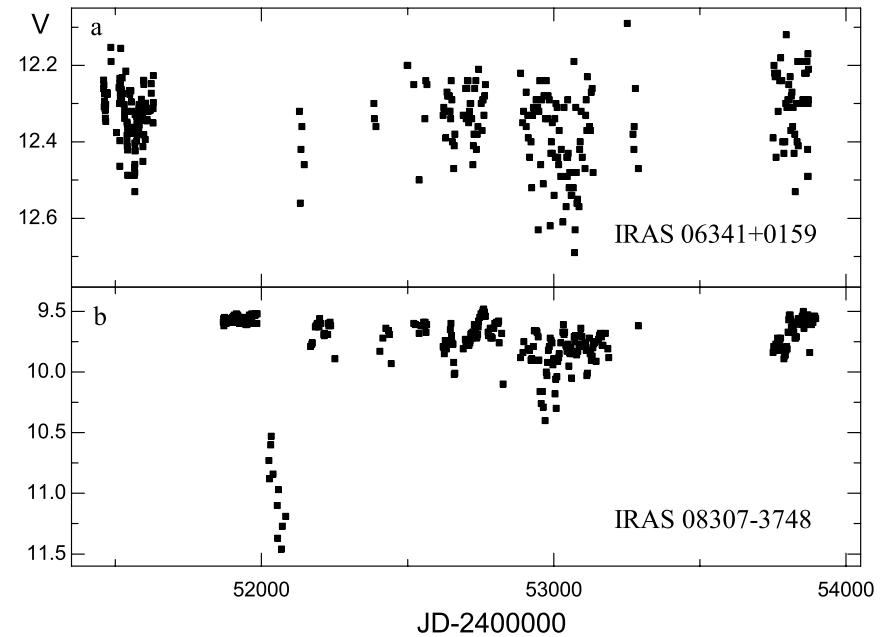

FIG. 11.-Light curves of strongly variable program objects. Top panel: The NSVS (earlier than JD 251600) and ASAS light curve of IRAS 06341+0159. Typical measurement uncertainties are 0.03-0.04 mag. Bottom panel: The ASAS light curve of IRAS $08307-3748=$ FX Vel. Typical measurement uncertainties are 0.01-0.02 mag near maximum and 0.03-0.05 mag near the deepest minimum.

De Winter et al. (2001) obtained an UBVR photometric observation in 1994 with $V=10.8$ mag that is over 1 mag fainter than Eggen's maximum brightness $(V=9.7 \mathrm{mag})$. At the same time, the All Sky Automated Survey (ASAS; Pojmanski 2002) light curve shows an irregular behavior (Fig. 11b) that looks similar to those of isolated Herbig Ae/Be stars with Algol-like photometric minima (Grinin et al. 1991). The nature of the variations is not clear as almost no color information is available. The data by de Winter et al. (2001) $U-B=-0.08 \mathrm{mag}$ and $B-V=0.18 \mathrm{mag}$, do not show a significant reddening, characteristic of the Algol-like minima. Nevertheless, the short-period eclipsing brightness variations can be ruled out, because the deepest minimum near JD 2,452,000 lasted for nearly 2 months.

FX Vel was identified with the IRAS source by Dong \& Hu (1991) but omitted in our previous selections of objects with the $\mathrm{B}[\mathrm{e}]$ phenomenon. Our spectrum (Fig. 12) shows the presence of forbidden lines. The double-peaked Balmer line profiles have the blue peak stronger than the red one. This is similar to classical Be stars and may indicate similar perturbations of the CS

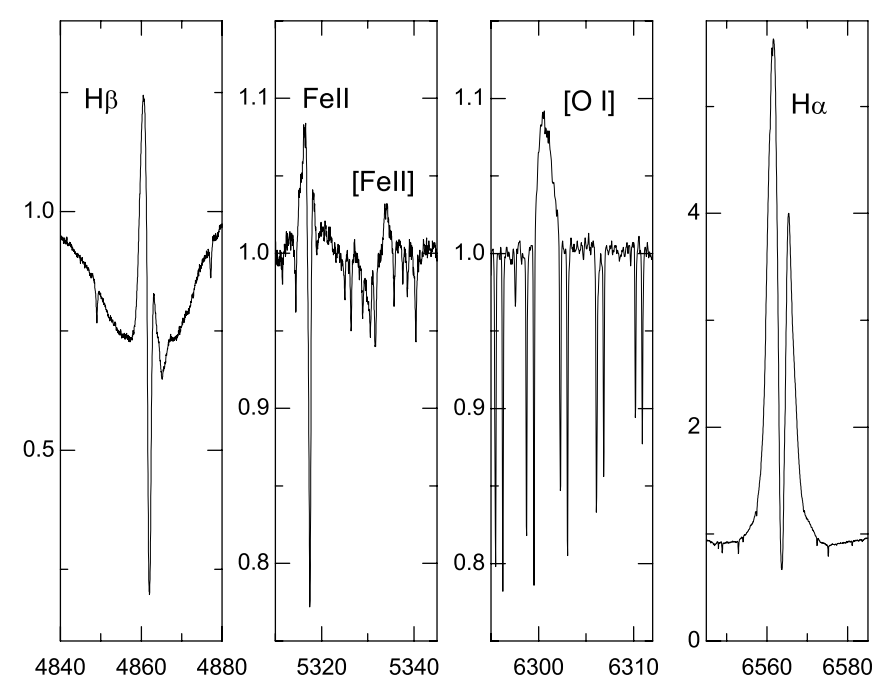

FIG. 12.-Portions of the optical high-resolution spectrum of IRAS 08307-3748. The spectrum was obtained at the $3.6 \mathrm{~m} \mathrm{CFHT}$ in 2004 December. Intensities and wavelengths are in the same units as in Fig. 3.

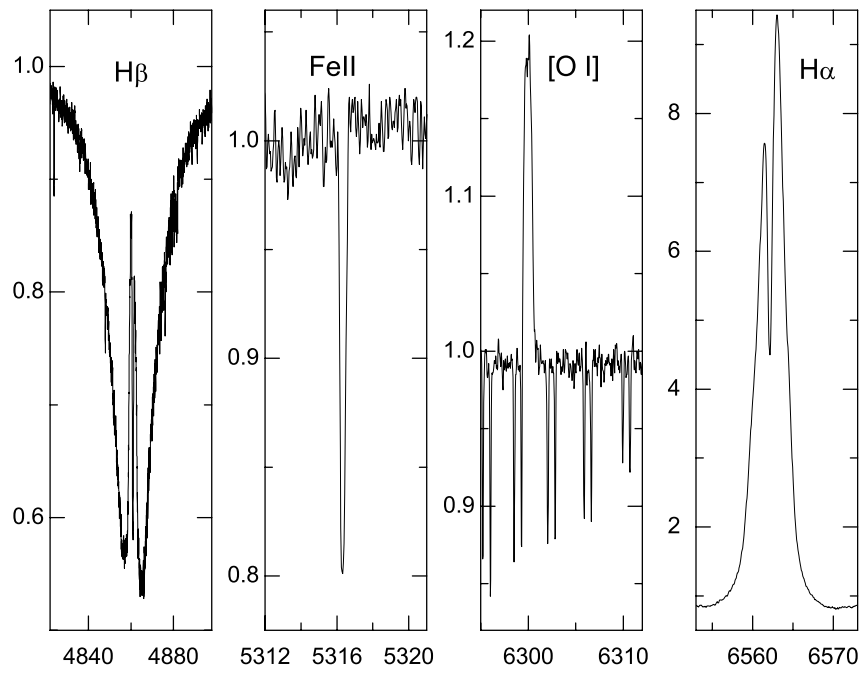

FIG. 13.- Portions of the optical high-resolution spectrum of IRAS 17449+2320. The spectrum was obtained at the $3.6 \mathrm{~m}$ CFHT in 2006 June. Intensities and wavelengths are in the same units as in Fig. 3.

disk density structure (Okazaki 1991). We also detected the weak $\mathrm{Li}$ I $6708 \AA$ and $\mathrm{Ca}$ I $6717 \AA$, similar to those of IRAS 03421+2935 (see Fig. 6).

\subsubsection{IRAS $17449+2320$}

The $\mathrm{H} \alpha$ emission was detected in the spectrum of this 10 mag star (also known as BD +23 3183) by Stephenson (1986). Downes \& Keyes (1988) obtained a low-resolution spectrum and described it as a Be star. Our spectrum of IRAS $17449+2320$ shows the Balmer and [O I] lines in emission, but Fe II lines in absorption (Fig. 13). The He I $4471 \AA$ line is very weak $(\mathrm{EW}=0.07 \AA)$ and is much weaker than the $\mathrm{Mg}$ II $4481 \AA$ line $(\mathrm{EW}=0.34 \AA)$. These features suggest a low $T_{\text {eff }}$ of the underlying star (early A-type).

Our optical photometry (Table 4) and the broad Balmer line wings (Fig. 13) indicate that the object is an unreddened A0 dwarf. Neglecting any CS contribution to the optical continuum, the distance toward it is $\sim 700$ pc (based on a calibration from Straizys \& Kuriliene 1981). Thus, this is one of the closest objects with the $\mathrm{B}[\mathrm{e}]$ phenomenon, most of which are located beyond $1 \mathrm{kpc}$ from the Sun (Miroshnichenko et al. 2006).

\subsection{Other Emission-Line/Large IR-Excess Objects}

The objects in the second group we report here have SEDs that are only similar to those of the objects with the $\mathrm{B}[\mathrm{e}]$ phenomenon in the IRAS wavelength range (see Fig. 14). Their near-IR colors (except for those of IRAS 17200-1355) are bluer, and they are located beyond the $\mathrm{B}[\mathrm{e}]$ objects area in Fig. 2. We would like to attract attention to them because of their unusual nearand mid-IR excesses. Also, our observations resulted in the detection of spectral line emission in most of these objects (see Fig. 15 and 16).

\subsubsection{IRAS $02013+7621$}

The optical counterpart of the IRAS source was found by Obayashi et al. (1998) projected onto the molecular cloud L1333. IRAS $02103+7621$ is one of five IRAS sources associated with $\mathrm{H} \alpha$ emission objects in this region. Kun et al. (2006) obtained a low-resolution $(R \sim 1000)$ spectrum of IRAS $02103+7621$, detected a Li I $6708 \AA$ absorption line in addition to a strong $\mathrm{H} \alpha$ line in emission, and classified the underlying star as $\mathrm{K} 7 \mathrm{~V}$.

Our data (Fig. 17) suggest an earlier spectral type of K4 V. The presence of the He I and He II lines in emission unambiguously 

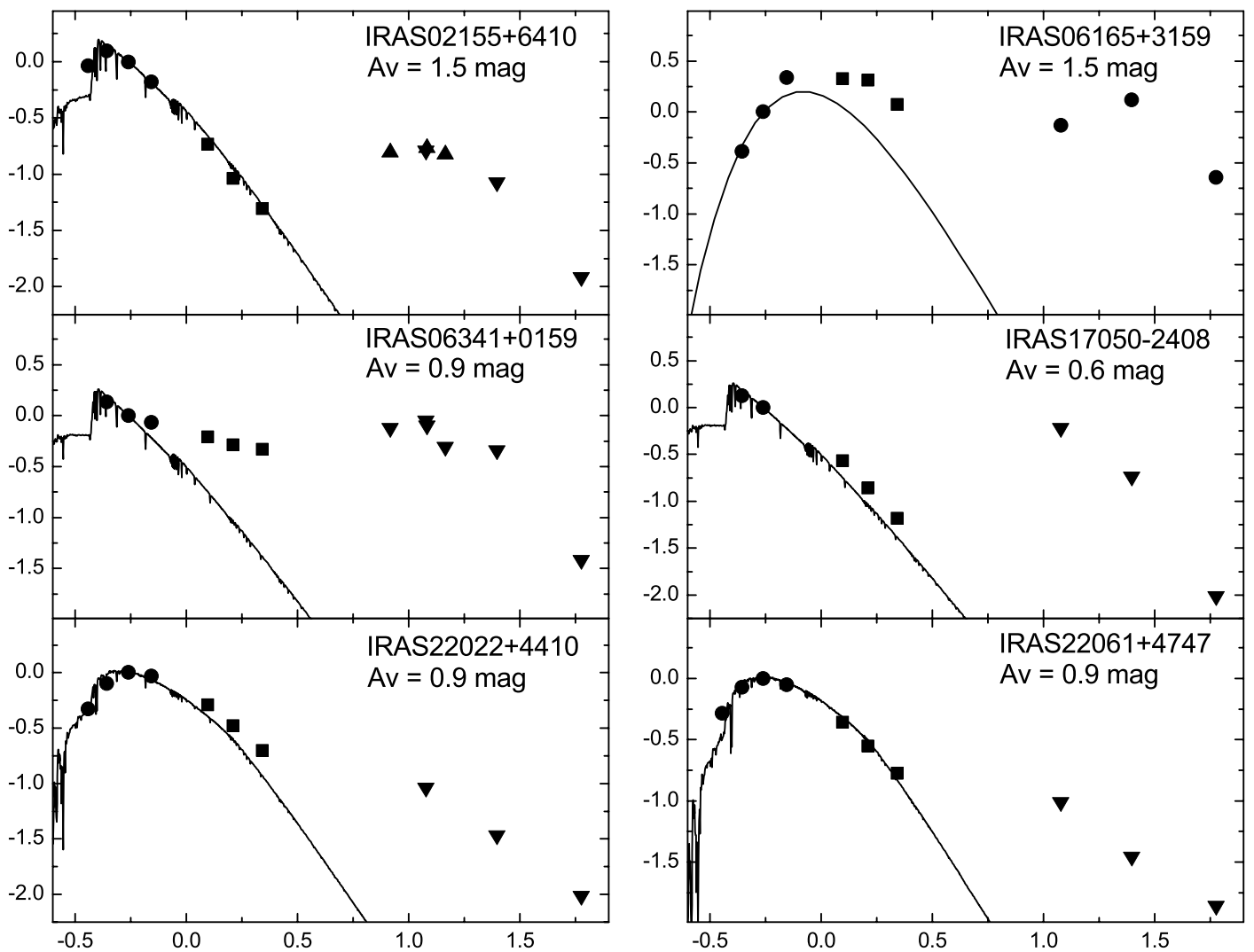

FIG. 14.- SEDs of the program objects without the $\mathrm{B}[\mathrm{e}]$ phenomenon. The units of fluxes and wavelengths, as well as the meaning of the symbols, are the same as in Fig. 20. The fluxes were dereddened using the interstellar extinction law from Savage \& Mathis (1979). The interstellar extinction $\left(A_{V}\right)$ for each object was derived from our spectral type estimates and available optical photometry. The solid lines represent Kurucz (1994) model atmospheres for the objects' spectral types $\left(T_{\text {eff }}=10,000 \mathrm{~K}\right.$ for IRAS $06341+0159 ; T_{\text {eff }}=9000 \mathrm{~K}$ for IRAS $02155+6410$ and IRAS $17050-2408 ; T_{\text {eff }}=6500 \mathrm{~K}$ for IRAS $22022+4410 ;$ and $T_{\text {eff }}=6000 \mathrm{~K}$ for IRAS $\left.22061+4747\right)$. A blackbody curve for $T=3500 \mathrm{~K}$ represents the stellar continuum for IRAS $06165+3158$. Only the objects with good optical photometry are shown.

indicates that the object is a pre-main-sequence T Tau star. Its IRAS fluxes are consistent with those of the coolest objects of this type.

\subsubsection{IRAS $02155+6410$}

IRAS $02155+6410$ was examined for the presence of the $\mathrm{SiO}$ maser emission (Jiang et al. 1999) and that of the ${ }^{12} \mathrm{CO}$ emis-



FIG. 15.-Portions of the optical low-resolution spectra obtained at the $0.8 \mathrm{~m}$ telescope of the Dark Sky Observatory. Intensities are corrected for the detector sensitivity and normalized at $5500 \AA$, and wavelengths are in $\AA$. sion (Kerton \& Brunt 2003), but none was detected. Our spectra show that the optical counterpart is a mildly reddened A3-type dwarf with no line emission, except for a slight asymmetry of the $\mathrm{H} \alpha$ profile (Fig. 15 and 16). It might be another member of a recently discovered, but not yet studied type of evolved early-type stars with CS dust (Miroshnichenko et al. 2003b, 2004).

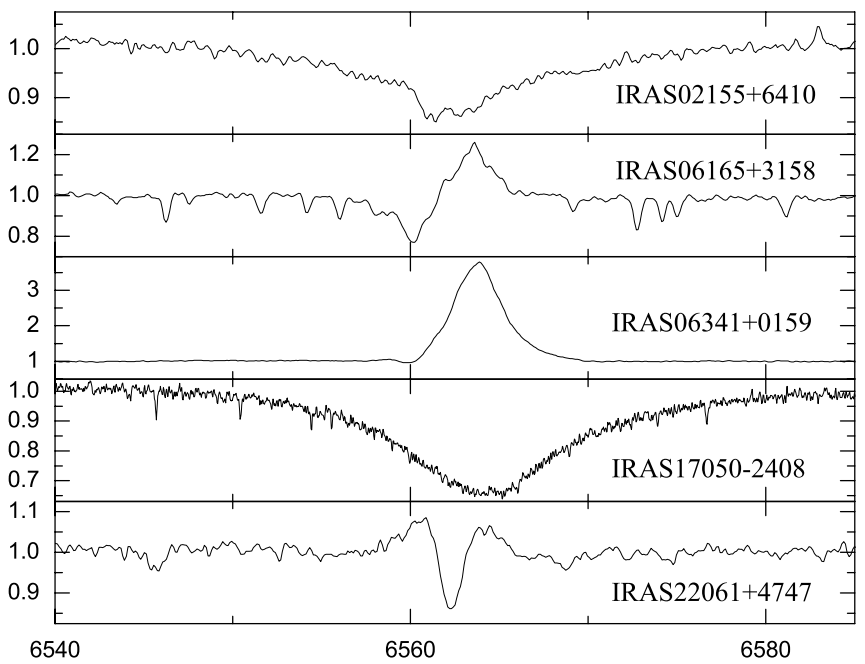

FIG. 16.-Portions of the optical high-resolution spectra obtained at the $3.6 \mathrm{~m}$ CFHT with the Gecko spectrograph. Intensities and wavelengths are in the same units as in Fig. 3. 


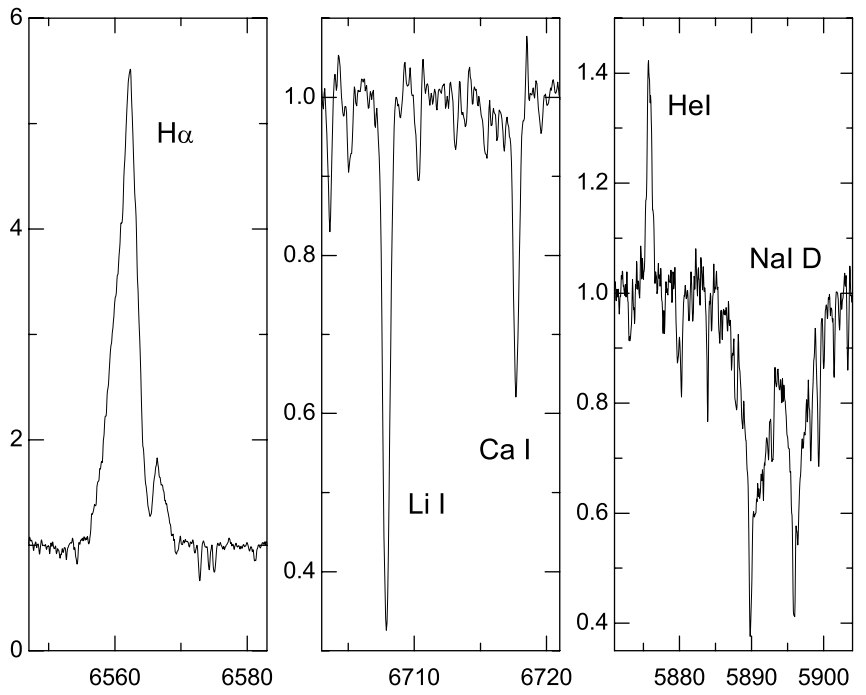

FIG. 17.- Portions of the optical high-resolution spectrum of IRAS $02103+$ 7621 obtained at the $2.7 \mathrm{~m}$ Harlan J. Smith Telescope of the McDonald Observatory (2005 December). Intensities and wavelengths are in the same units as in Fig. 3.

\subsubsection{IRAS $06165+3158$}

No OH maser emission at $1612 \mathrm{MHz}$ was detected from IRAS $06165+3158$ (Lewis et al. 1990). The only emission line seen in our spectra is $\mathrm{H} \alpha$, which has a P Cyg type profile (Fig. 16). The photospheric lines indicate a supergiant K-type spectrum, probably metal deficient. No Li I 6708 Å line or molecular bands are seen in the spectrum.

\subsubsection{IRAS $06341+0159$}

The positional separation of IRAS $06341+0159$ and its optical counterpart is $18^{\prime \prime}$, which is still within the IRAS position error box. The IRAS source has not been studied, while its optical counterpart was previously detected in the emission-line star surveys of Gonzalez \& Gonzalez (1954) and Kohoutek \& Wehmeyer (1999). Our optical photometry indicates that it is most likely a slightly reddened early-type star (see Fig. 14). Robotic telescope observations obtained in the course of the Northern Star Variability Survey (NSVS; Wozniak et al. 2004) and the ASAS survey (Pojmanski 2002) show significant variations with an amplitude of $\sim 0.5 \mathrm{mag}$ (Fig. 11a). The close brightness in the two data sets (the NSVS data are unfiltered, while the ASAS data are in the $V$ band) indirectly confirms our photometric data, showing small optical color-indices (see Table 4).

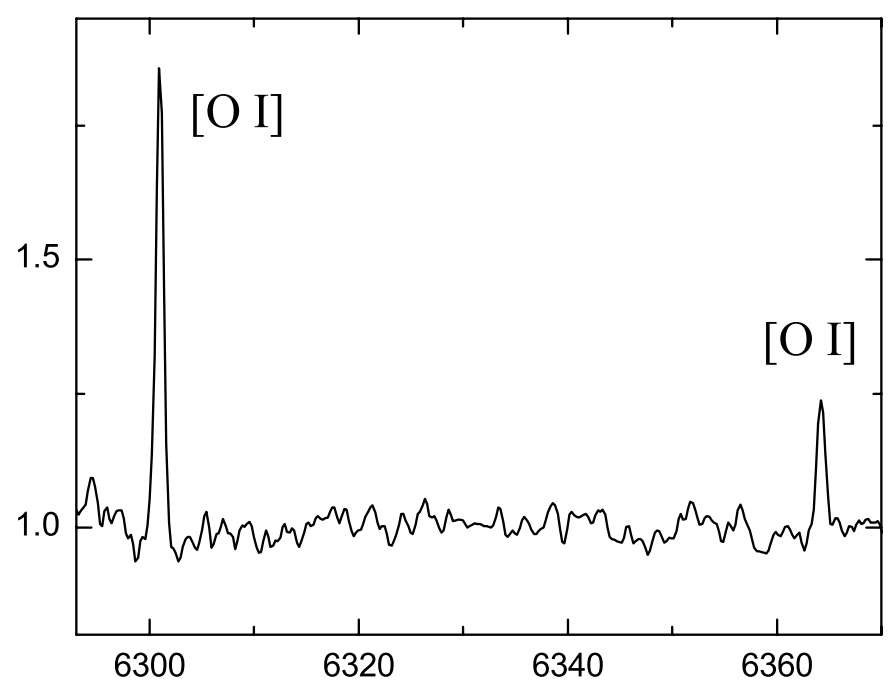

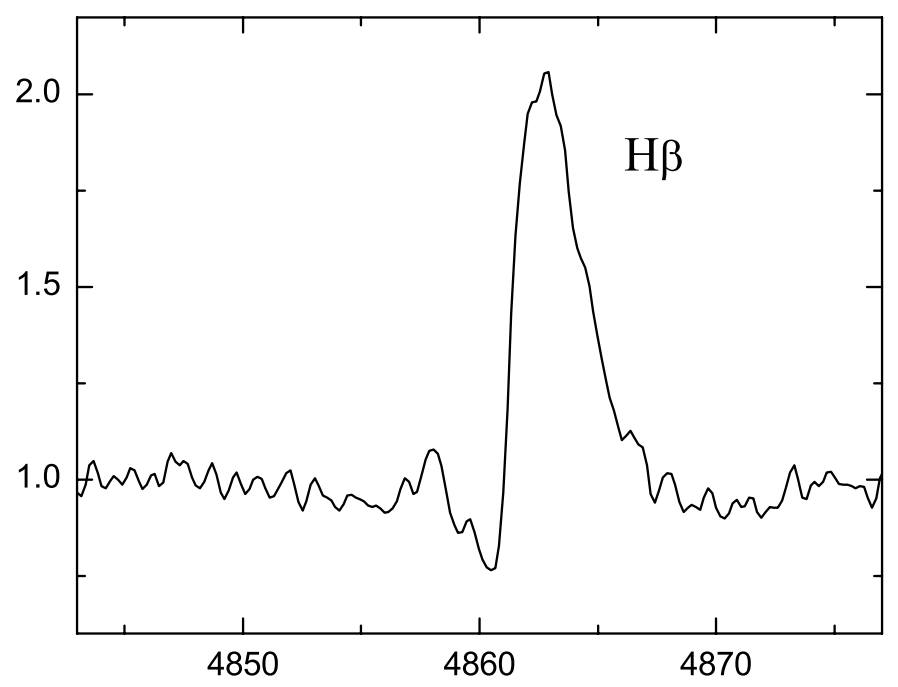

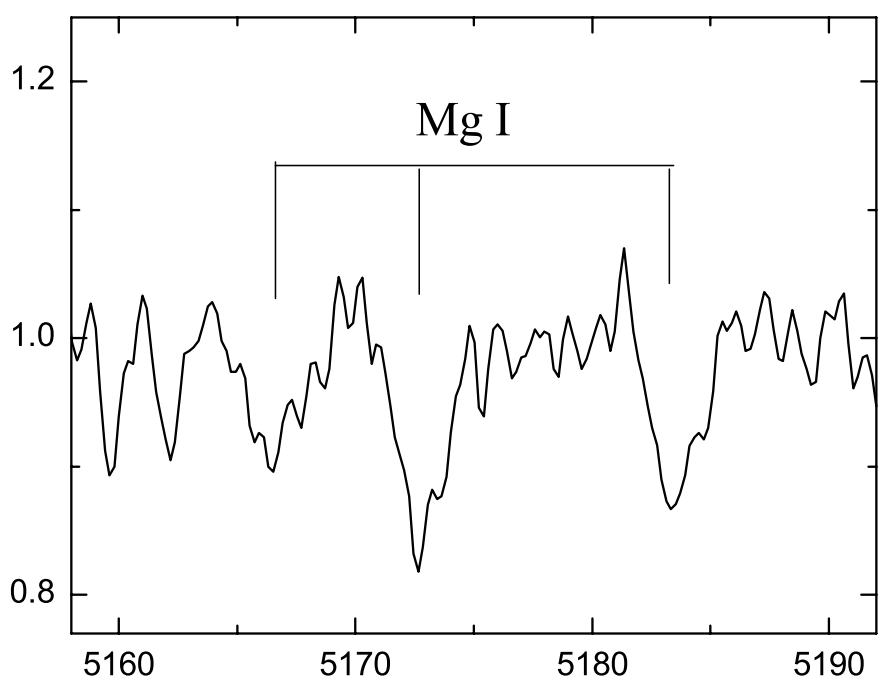

FIG. 18.- Portions of the optical high-resolution spectrum of IRAS 06341+0159 obtained at the $2.1 \mathrm{~m}$ telescope of the San Pedro Martir Observatory (2006 December). Intensities and wavelengths are in the same units as in Fig. 3. 

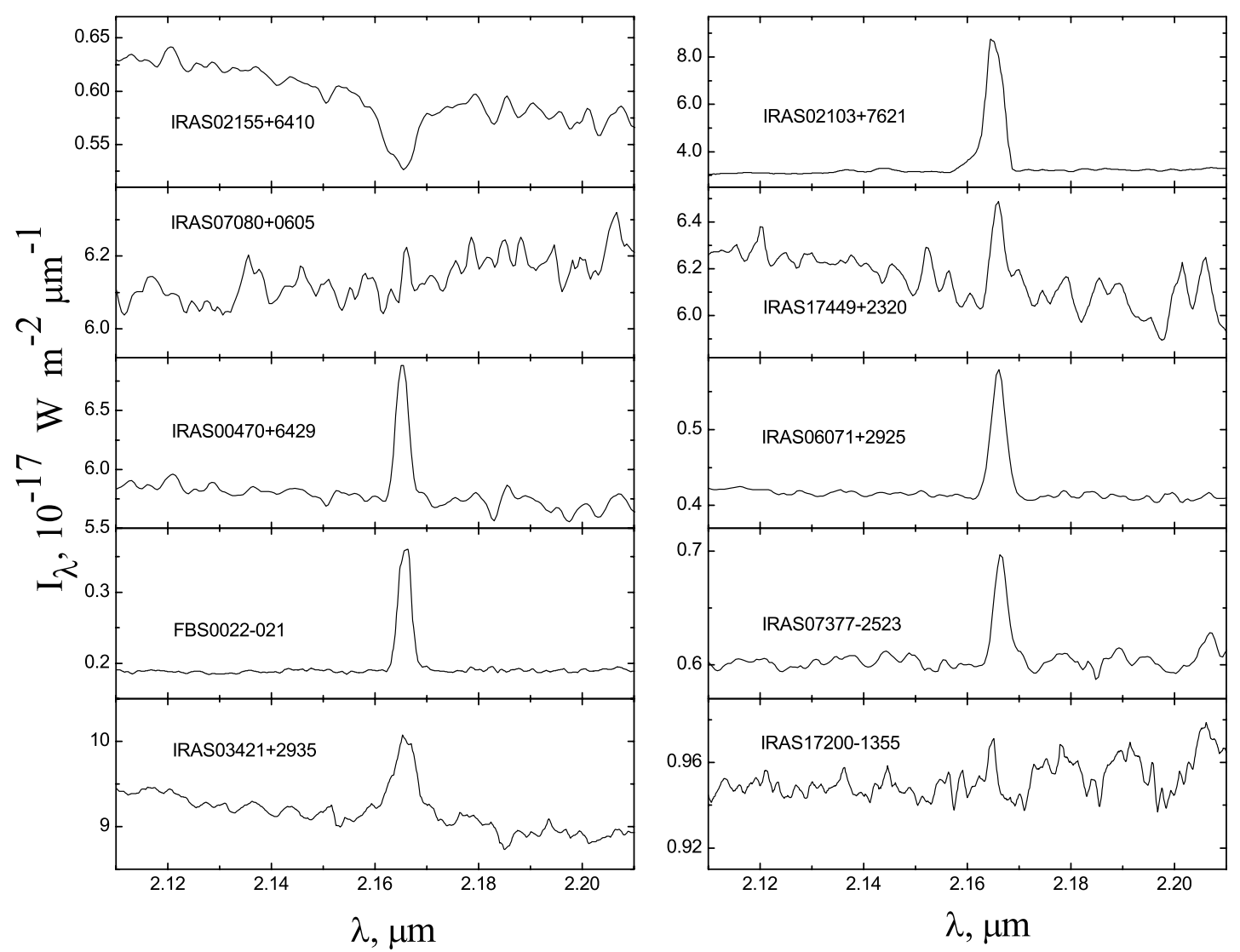

FIG. 19.- Portions of the near-IR low-resolution spectra obtained at the $3 \mathrm{~m}$ Shane Telescope of the Lick Observatory with the NIRI spectrograph. A region around the $\mathrm{Br} \gamma$ line is shown. Intensities (vertical axis) are in $10^{-17} \mathrm{~W} \mathrm{~m}^{-2} \mu \mathrm{m}^{-1}$, and wavelengths are in microns.

Our spectrum shows a strong $\mathrm{H} \alpha$ line on top of an almost featureless continuum. The line strength was found significantly different in 2003 (the CFHT spectrum; Fig. 16) and in 2005-2006 (it is almost the same in our two San Pedro Martir spectra obtained 14 months apart; Fig. 18).

$\mathrm{H} \beta$ is in also emission, while $\mathrm{H} \gamma$ and $\mathrm{H} \delta$ are in absorption. The He I $5876 \AA$ line is seen in absorption next to a strong Na I D doublet. We detected no Fe II lines, but the [O I] 6300 and $6363 \AA$ lines are clearly seen in emission. Both spectra obtained at San Pedro Martir show the Mg I absorption triplet at 5167, 5172, and $5183 \AA$ typical of late-type stars (Fig. 18). In addition, the Li I $6708 \AA$ absorption line can be marginally recognized. Although the signal-to-noise ratio in our spectra $(\sim 40)$ was high enough to detect other photospheric absorptions expected in the yellow-red part of the spectrum, they are not clearly seen.

The observed features most likely represent a composite spectrum of a binary system, which contains a hot and a cool companion. The hot companion is probably brighter than the cool one to account for the presence of only a few late-type star features. Its fast rotation can account for the absence of the early-type star features. The CS continuum emission may also contribute to the spectral line veiling.

\subsubsection{IRAS $17050-2408$}

The optical counterpart of IRAS 17050-2408 is an almost unreddened A-type star (HD 154722, A2 IV; Houk \& SmithMoore 1988). te Lintel Hekkert et al. (1991) did not detect any $\mathrm{OH}$ maser emission from this source, whose IRAS colors are typical for post-AGB objects. Kwok et al. (1997) reported the presence of the $9.7 \mu \mathrm{m}$ silicate band in emission in the object's IRAS LRS spectrum. Our optical spectrum in the $\mathrm{H} \alpha$ region shows no emission in the line (Fig. 16), whose profile is consistent with the mentioned spectral classification. The star is located in the direction of the star-forming clouds in Ophiuchus, but its decreasing IR flux toward longer wavelengths is not typical for young main-sequence Vega-type objects. Its nature might be similar to that of IRAS $02155+6410$.

\subsubsection{IRAS 17200-1355}

In the only paper that studied IRAS $17200-1355$ along with a broad selection of other IRAS sources (Carballo et al. 1992), it was listed as an evolved Galactic object with no comment on its nature. Our flux calibrated spectrum (Fig. 19) clearly shows only two strong emission lines ( $\mathrm{H} \alpha$ and $\left.\mathrm{He}_{\mathrm{I}} 1.083 \mu \mathrm{m}\right)$. The SED suggests an early spectral type with a relatively high reddening ( $A_{V} \sim 3 \mathrm{mag}$ ), which is not strong enough to eliminate the IR excess. The object satisfies the photometric criteria for stars with the $\mathrm{B}[\mathrm{e}]$ phenomenon, but forbidden lines are not clearly seen in the spectrum. The absence of Fe II lines may be due to low $T_{\text {eff }}$, while the absence of [ $\left.\mathrm{O}_{\mathrm{I}}\right]$ lines may be due to the combination of their weakness and our low resolution. It can be considered as $\mathrm{B}[\mathrm{e}]$ candidate object and needs further higher resolution data.

\subsubsection{IRAS $20422+4644$}

The IRAS images show IRAS $20422+4644$ as a pointlike source with fluxes that are large enough to be detected by the IRAS LRS (see Table 3). Kwok et al. (1997) classified the IRAS LRS spectrum as unusual (type "U"), although it is noisy with mostly flat continuum. The large IRAS fluxes are confirmed by the Mid-Course Space Experiment (MSX) data (Egan et al. 2003). The optical counterpart seems to be very reddened (the USNO-B1.0 $B-R$ color index is $\sim 2.5 \mathrm{mag}$ ). Our spectrum (Fig. 15) shows a noticeably 

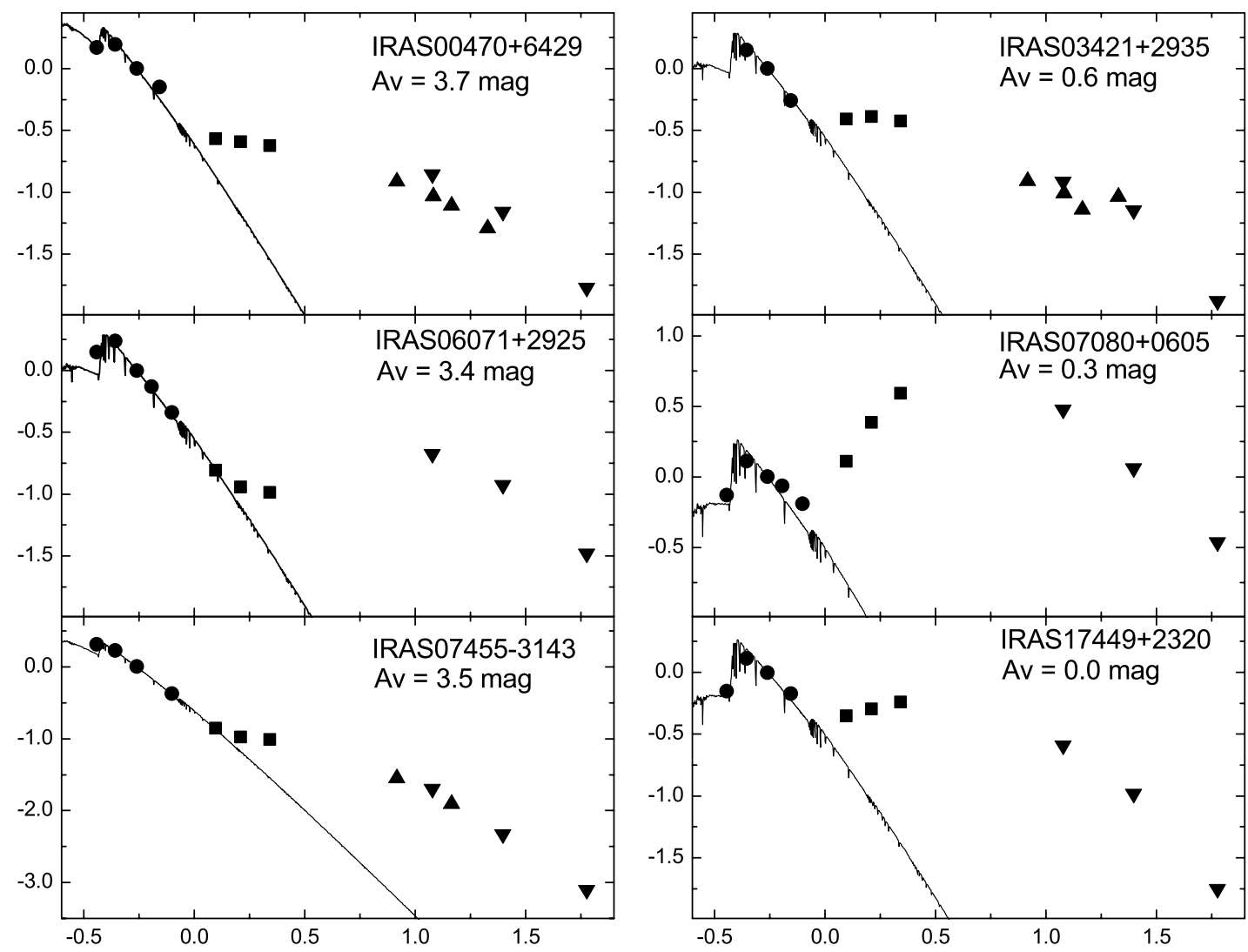

FIG. 20.- SEDs of the program objects with the B[e] phenomenon. Logarithm of the flux normalized to that in the $V$ band (vertical axis) is plotted vs. logarithm of the wavelength in microns. The fluxes were dereddened using the interstellar extinction law from Savage \& Mathis $(1979)$. The interstellar extinction $\left(A_{V}\right)$ for each object was derived from our spectral type estimates and available optical photometry. Symbols: optical photometry, circles; near-IR photometry (mostly from 2MASS), squares; MSX data, upward triangles; and IRAS data, downward triangles. The solid lines represent Kurucz (1994) model atmospheres for the objects' spectral types $\left(T_{\text {eff }}=10,000 \mathrm{~K}\right.$ for IRAS $07080+0605$, and IRAS $17449+2320 ; T_{\text {eff }}=12,000 \mathrm{~K}$ for IRAS $03421+2935$ and IRAS $06071+2925$; and $T_{\text {eff }}=16,000 \mathrm{~K}$ for IRAS $00470+6429$ and IRAS $07455-3143$ ).

reddened continuum with absorption lines, typical of an earlytype star. The narrow Balmer lines suggest a high luminosity (A0 Ib).

\subsubsection{IRAS $22022+4410$ and IRAS $22061+4747$}

These two unstudied IRAS sources are associated with $10 \mathrm{mag}$ stars. IRAS $22022+4410$ has a spectral type F7 III, while IRAS $22061+4747(=\mathrm{BD}+473700)$ is a later, G3 III-IV, star (Fig. 15) that is probably metal-deficient. We do not have a high-resolution $\mathrm{H} \alpha$ profile of the former, but the latter shows a weak doublepeaked emission (Fig. 16). Both sources are mildly reddened $\left(A_{V} \sim 1\right.$ mag; see Table 4$)$.

\section{DISCUSSION}

None of the newly found objects with the $\mathrm{B}[\mathrm{e}]$ phenomenon belongs to a star-forming region. Also none shows features of the post-AGB objects (molecular line emission, [O III] line emission, extended nebulae). They also exhibit no evidence for high luminosity. The only object with an uncertain luminosity is FBS $0022-021$, but it is very unusual for a high-luminosity star to be at such a high Galactic latitude. Therefore, all nine objects should be considered FS CMa stars.

Like the previously known FS CMa stars, the newly found group members show very little difference between the IRAS and MSX fluxes obtained 13 years apart. This indicates stability of their dusty environments on the timescale of years and confirms the initial IRAS detections at the higher positional accuracy of MSX. Also, our simultaneous optical and IR spectroscopy at Lick confirms the optical and near-IR identifications and show line emission in the near-IR region (Fig. 19).

The results of our catalog search and observations suggest new photometric criteria for stars with the $\mathrm{B}[\mathrm{e}]$ phenomenon, especially for FS CMa stars. In addition to a specific SED in the mid-IR region (Fig. 1, dashed box), they all have $12 \mu \mathrm{m} I R A S$ fluxes greater than the $K$-band fluxes and the $J-K$ color indices greater than $\sim 1.3 \mathrm{mag}$. The latter two criteria are almost reddening-independent and constrain the SED all the way to the short-wavelength boundary of the IR excess due to CS dust $(\lambda=1-1.5 \mu \mathrm{m}$; see Fig. 20). These properties can be important in further attempts to search for more such objects. They could still be found among the IRAS PSC sources with low-quality $60 \mu \mathrm{m}$ fluxes.

Our spectroscopic data revealed a new feature of the FS CMa star group. The $\mathrm{B}[\mathrm{e}]$ phenomenon can be observed even in lower luminosity and temperature objects than originally thought. There are only two A-type objects in the original list of $\mathrm{B}[\mathrm{e}]$ stars by Allen \& Swings (1976): HD 190073 (A0 Ve) and 3 Pup (A2 Iab:e). We found at least four more A-type objects: IRAS 07080+0605, IRAS 07455-3143, IRAS 08307-3748, and IRAS 17449+2320. This improves the expectations for finding more potential group members among objects with lower optical and IR fluxes.

At lower temperatures, $\mathrm{Fe}$ II lines may be seen mostly in absorption and $\left[\mathrm{O}_{\mathrm{I}}\right]$ lines may be very weak. Thus high-resolution observations with high signal-to-noise ratios in the continuum are important for detecting such FS CMa objects. Also photometric criteria will play a crucial role at the stage of initial candidate identification. 
Three of the nine newly found FS CMa stars show direct evidence for the presence of the secondary companions (IRAS 03421+ $2935=$ MWC 728, IRAS 07455-3143 = AS 174, and IRAS 08307-3748 = FX Vel). The unusually strong line emission and IR excesses of the others suggest their presence indirectly (see $\S 3$ ). This gives further support to our hypothesis that FS CMa stars are binary systems (Miroshnichenko et al. 2006). On the other hand, binarity implies variations of the radial velocity, which is a good indicator of the objects' distances. The latter are hard to determine from the spectral line intensities and intensity ratios because of the uncertain amount of CS veiling and relative brightness of the companions. Therefore, multiple spectra need to be obtained to constrain fundamental and orbital parameters of the systems.

All but two (IRAS $02103+7621$ and IRAS $06165+3158$ ) of the objects with no $\mathrm{B}[\mathrm{e}]$ phenomenon are early-type stars with strong IR excesses. Some of them show signs of evolution beyond the main-sequence stage in their luminosity types. They neither clearly belong to star-forming regions nor show signs of postAGB evolution. It is hard to believe that their IR excesses are due to dust that survived from the protostellar stage, as in Vega-like stars. The latter show no near-IR excess, but just a far-IR excess due to cold dust. Our objects show the opposite, the presence of a near-IR excess and a sharp decrease of the IR flux longward of 10-30 $\mu \mathrm{m}$.

Our current hypothesis about their nature is that dust formation is possible as the star expands on the way toward the red giant evolutionary stage. A few years ago we found two such objects, HD 19993 (Miroshnichenko et al. 2003b) and HD 35929 (Miroshnichenko et al. 2004). Our new findings suggest that the phenomenon occurs more frequently than just by chance.

It cannot be excluded that all these objects with no $\mathrm{B}[\mathrm{e}]$ phenomenon are binary systems, just as the FS CMa stars, but with different orbital parameters and perhaps at a different stage of evolution. However, our spectroscopic data show no evidence for the presence of the secondary companions.

\section{CONCLUSIONS}

Our search in the IRAS PSC resulted in finding nine new objects showing the $\mathrm{B}[\mathrm{e}]$ phenomenon. None of them shows clear signs of high luminosity, and three of them exhibit direct evidence for binarity. They all can be considered to be FS CMa stars. Therefore, the total number of objects in this group reaches 40 , and the group becomes the largest among the family of dust-forming hot stars.

Our newly found FS CMa stars show that the $\mathrm{B}[\mathrm{e}]$ phenomenon can be observed at lower $T_{\text {eff }}$ (spectral type A) and lower luminos- ities than thought before. This is an encouraging result, suggesting that more such objects can be found in the IRAS PSC. Since their IR fluxes steeply decrease toward longer wavelengths, they might have uncertain or even undetected $60 \mu \mathrm{m}$ fluxes. Therefore, our photometric criteria presented in this paper may be used to search for them.

We have also found a few early-type objects with no signs for the $\mathrm{B}[\mathrm{e}]$ phenomenon, but with strong IR excesses. These may be evolved stars or binary systems that form CS dust at an evolutionary phase between the main sequence and the red giant stage. This result amplifies our previous findings of two giants with similar properties. Dust formation in such objects has not been explored yet. Another interesting result is the finding of the two dusty high-luminosity objects (IRAS $06165+3158$ and IRAS $20422+4644)$, which are very rare and whose evolution is still poorly known.

We are going to continue observing the presented objects, especially the FS CMa stars, and will present a more detailed analysis of individual objects as well as small groups of them, including still poorly studied other FS CMa stars (Miroshnichenko 2007), in the future papers of this series.

A. M. and K. S. B. acknowledge support from NASA grant NAG5-8054 at the early stage of this research. At later stages, the research was supported by a Civilian Research and Development Foundation (CRDF) through the grant RUP1-2687-NA-05 to K. S. B., V. G. K., A. M., and E. L. C.; A. M. also acknowledges support from the UNCG New Faculty Grant 331511. This research has made use of the SIMBAD database operated at CDS, Strasbourg, France. This publication makes use of data products from the Two Micron All Sky Survey, which is a joint project of the University of Massachusetts and the Infrared Processing and Analysis Center/California Institute of Technology, funded by the National Aeronautics and Space Administration and the National Science Foundation. Data from the USNO-B1.0 cata$\log$ and from the NSVS and ASAS databases were used in our research. This publication is partially based on observations obtained at the Canada-France-Hawaii Telescope (CFHT), which is operated by the National Research Council of Canada, the Institut National des Sciences de l'Univers of the Centre National de la Recherche Scientifique of France, and the University of Hawaii. The ESPaDOnS spectra obtained at CFHT were reduced using Libre-ESpRIT, written by J.-F. Donati from Observatoire Midi-Pyrenees, and provided by CFHT. We thank the anonymous referee for the suggestions that improved the material presentation.
Allen, D. A., \& Swings, J.-P. 1976, A\&A, 47, 293

Baudrand, J., \& Vitry, R. 2000, Proc. SPIE, 4008, 182

Carballo, R., Wesselius, P. R., \& Whittet, D. C. B. 1992, A\&A, 262, 106 Cazzolato, F., \& Pineault, S. 2003, AJ, 125, 2050

Chengalur, J. N., Lewis, B. M., Eder, J., \& Terzian, Y. 1993, ApJS, 89, 189 Coyne, G. V., \& MacConnel, D. J., 1983, Vatican Observ. Publ., 2, 73

Cutri, R. M., et al. 2003, CDS/ADC Collection of Electronic Catalogues, 2246

de Winter, D., van den Ancker, M. E., Maira, A., Thé, P. S., Tjin A Dje, H. R. E., Redondo, I., Eiroa, C., \& Molster, F. J. 2001, A\&A, 380, 609

Dolidze, N., \& Ponomareva, G. 1975, Soviet Astron., 9, 157

Donati, J.-S., Semel, M., Carter, B. D., Rees, D. E., \& Collier Cameron, A. 1997, MNRAS, 291, 658

Dong, Y. S., \& Hu, J. Y. 1991, Chinese A\&A, 15, 275

Downes, R. A., \& Keyes, C. D. 1988, AJ, 96, 777

Downes, R. A., Webbink, R. F., Shara, M. M., Ritter, H., Kolb, U., \& Duerbeck, H. W. 2001, PASP, 113, 764

\section{REFERENCES}

Egan, M. P., et al. 2003, The Midcourse Space Experiment Point Source Catalog, ver. 2.3 (Air Force Res. Lab.Tech. Rep. AFRL-VS-TR-2003-1589; Hanscom AFB: AFRL)

Eggen, O. Y. 1978, AJ, 83, 288

Gonzalez, G., \& Gonzalez, G. 1954, Bol. Obs. Tonantz. Tacub., 1, No 9, 3

Gray, R. O., Corbally, C. J., Garrison, R. F., McFadden, M. T., \& Robinson, P. E. 2003, AJ, 126, 2048

Grinin, V. P., Kiselev, N. N., Chernova, G. P., Minikulov, N. Kh., \& Voshchinnikov, N. V. 1991, Ap\&SS, 186, 283

Gualandi, R., \& Merighi, R. 2001, BFOSC-Bologna Faint Object Spectrograph and Camera, Manuale Utente Rel. 2.0

Henize, K. G. 1976, ApJS, 30, 491

Hoard, D. W., Wachter, S., Clark, L. L., \& Bowers, T. P. 2002, ApJ, 565, 511 Hog, E., et al. 2000, A\&A, 355, L27

Houk, N., \& Smith-Moore, M. 1988, Catalogue of Two-dimensional Spectral Types for the HD Stars (Michigan Spectral Survey, Vol. 4; Ann Arbor: Univ. Michigan) 
Jiang, B. W., Deguchi, S., \& Ramesh, B. 1999, PASJ, 51, 95

Kelly, D. M., \& Hrivnak, B. J. 2005, ApJ, 629, 1040

Kerton, C. R., \& Brunt, C. M. 2003, A\&A, 399, 1083

Kohoutek, L., \& Wehmeyer, H. 1999, A\&AS, 134, 255

Kopylov, I. M., Leushin, V. V., Topilskaya, G. P., Tsymbal, V. V., \& Gvozd, Y. A. 1989, Bull. Special Astrof. Obs. North Caucasus, 28, 67

Kopylov, I. M., Lipovetsky, V. A., Somov, N. N., Somova, T. A., \& Stepanian, J. A. 1988, Astrofizika, 28, 287

Kukarkin, B. V., Kholopov, P. N., Kukarkina, N. P., \& Perova, N. B. 1972, Inf. Bull. Variable Stars, 717, 1

Kun, M., Nikolić, S., Johansson, L. E. B., Balog, Z., \& Gáspár, A. 2006, MNRAS, 371, 732

Kurucz, R. L. 1994, Kurucz CD-ROM 19, Solar Model Abundance Model Atmospheres (Cambridge: SAO)

Kwok, S., Volk, K., \& Bidelman, W. P. 1997, ApJS, 112, 557

Lamers, H. J. G. L. M., Zickgraf, F.-J., de Winter, D., Houziaux, L., \& Zorec, J. 1998, A\&A, 340, 117

Levine, S., \& Chakrabarty, D. 1995, IA-UNAM Tech. Rep., MU-94-04

Lewis, B. M., Eder, J., \& Terzian, Y. 1990, ApJ, 362, 634

Lloyd Evans, T. 1985, MNRAS, 217, 493

Malkov, O. Y., Oblak, E., Snegireva, E. A., \& Torra, J. 2006, A\&A, 446, 785

Manset, N., \& Donati, J.-F. 2003, Proc. SPIE, 4843, 425

Markarian, B. E., Lipovetsky, V. A., Stepanian, J. A., Erastova, L. K., \& Shapovalova, A. I. 1989, Soobscheniya. Spec. Astrophys. Observ., 62, 5

McCarthy, J. K., Sandiford, B. A., Boyd, D., \& Booth, J. 1993, PASP, 105, 881

Meixner, M., et al. 1999, ApJS, 122, 221

Merrill, P. M., \& Burwell, C. G. 1949, ApJ, 110, 387 1950, ApJ, 112, 72

Miroshnichenko, A. S. 2007, ApJ, 667, 497

Miroshnichenko, A. S., Bjorkman, K. S., Chentsov, E. L., \& Klochkova, V. G. 2002, in ASP Conf. Ser. 279, Exotic Stars as Challenges to Stellar Evolution, ed. C. A. Tout, \& W. Van Hamme, (San Francisco: ASP), 303

Miroshnichenko, A. S., Gray, R. O., Klochkova, V. G., Bjorkman, K. S., \& Kuratov, K. S. 2004, A\&A, 427, 937
Miroshnichenko, A. S., et al. 2000, A\&AS, 147, 5 2003a, BAAS, 35, 1359

2003b, A\&A, 412, 219

2006, in ASP Conf. Ser. 355, Stars with the B[e] Phenomenon, ed. M.

Kraus, \& A. S. Miroshnichenko (San Francisco: ASP), 315

Monet, D. G., et al. 2003, AJ, 125, 984

Obayashi, A., Kun, M., Sato, F., Yonekura, Y., \& Fukui, Y. 1998, AJ, 115, 274

Okazaki, A. T. 1991, PASJ, 43, 75

Orsatti, A. M. 1992, AJ, 104, 590

Panchuk, V. E., Klochkova, V. G., \& Najdenov, I. D. 1999, preprint (Special Astrophys. Observ. 135)

Parthasarathy, M., Vijapukar, J., \& Drilling, J. S. 2000, A\&AS, 145, 269

Pojmanski, G. 2002, Acta Astron., 52, 397

Reed, B. C. 1998, ApJS, 115, 271

Rudy, R. J., Puetter, R. C., \& Mazuk, S. 1999, AJ, 118, 666

Savage, B. D., \& Mathis, J. S. 1979, ARA\&A, 17, 73

Sheikina, T. A., Miroshnichenko, A. S., \& Corporon, P. 2000, in ASP Conf. Ser. 214, The Be Phenomenon in Early-Type Stars, ed. M. A. Smith, H. F. Henrichs, \& J. Fabregat (San Francisco: ASP), 494

Stephenson, C. B. 1986, ApJ, 300, 779

Stephenson, C. B., \& Sanduleak, N. 1971, Publ. Warner and Swassey Obs., 1, No.1

Straizys, V., \& Kuriliene, G. 1981, Ap\&SS, 80, 353

Strohmeier, W., Ott, H., \& Schoffel, E. 1968, Inf. Bull. Variable Stars, 261, 1 te Lintel Hekkert, P., Caswell, J. L., Habing, H. J., Haynes, R. F., \& Norris, R. P. 1991, A\&AS, 90, 327

Tull, R. G., MacQueen, P. J., Sneden, C., \& Lambert, D. L. 1995, PASP, 107, 251

Vieira, S. L. A., Corradi, W. J. B., Alencar, S. H. P., Mendes, L. T. S., Torres, C. A. O., Quast, G. R., Guimaraes, M. M., \& Da Silva, L. 2003, AJ, 126, 2971

Wozniak, P. R., et al. 2004, AJ, 127, 2436

Zharikov, S., Tovmassian, G., \& Costero, R. 2004, Rev. Mex. A\&A Conf. Ser., 20,44 


\section{AUTHOR QUERIES}

Q1 au: Your article has been edited for grammar, consistency, and to conform to journal style (see Instructions to Authors). To expedite publication, we generally do not query every routine grammatical and style change made to a manuscript, although all substantive changes have been noted. Please review the article carefully. Note that we may be unable to make changes that conflict with journal style, obscure meaning, or create grammatical or other problems. Also note that article proofs via PDF do not show corrections that will have been made by the typesetter's proofreader, which generally include corrections of errors of page layout, figure placement, and spacing and font mistakes; feel free to mark any errors you notice in these areas. When sending us your corrections, if you are writing them by hand, please print clearly. If sending a fax, please do not write too close to the margins of the page, as these are often cut off in fax transmission. Finally, please note that a delayed, incomplete, or illegible response may delay the publication of your article while we contact you.

Q2 au: Ordinarily, unless there is a specific reason to do so, we do not put catalogs in the reference section. I have put that information in a footnote instead. Okay?

Q3 au: ApJ capitalized de, von, van, etc., in names at the begininning of sentences, hence "De Winter here. However, I have left "te Lintel Hekkert" in two places, because I think to capitalize it would misrepresent the name.

Q4 au: Figures need to be cited sequentially in the paper (that is, at some point Fig. 9, for example, must be cited between Fig. 8 and Fig. 10; as long as this occurs, it doesn't matter if Fig. 10 is cited earlier, before Fig. 9). To accomplish this, I have renumbered most of the figures in the paper from this point on 